\title{
Iterative Analysis of the Unique Positive Solution for a Class of Singular Nonlinear Boundary Value Problems Involving Two Types of Fractional Derivatives with $p$-Laplacian Operator
}

\author{
Fang Wang $\mathbb{D}^{1},{ }^{1}$ Lishan Liu $\mathbb{D}^{1},{ }^{1,2}$ Yonghong $W u\left(\mathbb{D},{ }^{2}\right.$ and Yumei Zou $\mathbb{i}^{3}$ \\ ${ }^{1}$ School of Mathematical Sciences, Qufu Normal University, Qufu 273165, Shandong, China \\ ${ }^{2}$ Department of Mathematics and Statistics, Curtin University, Perth, WA 6845, Australia \\ ${ }^{3}$ Department of Statistics and Finance, Shandong University of Science and Technology, Qingdao 266590, China
}

Correspondence should be addressed to Lishan Liu; mathlls@163.com

Received 4 April 2019; Revised 24 August 2019; Accepted 30 August 2019; Published 27 October 2019

Academic Editor: Sergey Dashkovskiy

Copyright (c) 2019 Fang Wang et al. This is an open access article distributed under the Creative Commons Attribution License, which permits unrestricted use, distribution, and reproduction in any medium, provided the original work is properly cited.

\begin{abstract}
This article is concerned with a class of singular nonlinear fractional boundary value problems with $p$-Laplacian operator, which contains Riemann-Liouville fractional derivative and Caputo fractional derivative. The boundary conditions are made up of two kinds of Riemann-Stieltjes integral boundary conditions and nonlocal infinite-point boundary conditions, and different fractional orders are involved in the boundary conditions and the nonlinear term, respectively. Based on the method of reducing the order of fractional derivative, some properties of the corresponding Green's function, and the fixed point theorem of mixed monotone operator, an interesting result on the iterative sequence of the uniqueness of positive solutions is obtained under the assumption that the nonlinear term may be singular in regard to both the time variable and space variables. And we obtain the dependence of solution upon parameter. Furthermore, two numerical examples are presented to illustrate the application of our main results.
\end{abstract}

\section{Introduction}

In the past decades, fractional differential equations arise in many mathematical disciplines as the analogue modeling of systems and processes in many scientific fields, such as control theory and engineering. In fact, fractional-order models have proved to be valuable tools in modeling many physical phenomena (for details, see [1-3] and the references therein). Accordingly, there has been a substantial development in the research for fractional differential equations, the properties of solutions, especially. We refer the readers to the papers [454]. For instance, in [15], $\mathrm{Xu}$ and Wei investigated the positive solutions of the following fractional differential equations:

$$
\left\{\begin{array}{l}
D_{0+}^{\alpha}\left(D_{0+}^{\alpha} x(t)\right)=f\left(t, x(t), x^{\prime}(t),-D_{0+}^{\alpha} x(t)\right), \quad t \in[0,1], \\
x(0)=x^{\prime}(0)=x^{\prime}(1)=0, \\
D_{0+}^{\alpha} x(0)=D_{0+}^{\alpha+1} x(0)=D_{0+}^{\alpha+1} x(1)=0,
\end{array}\right.
$$

where $D_{0+}^{\alpha}$ is the Riemann-Liouville's fractional derivative, $2<\alpha \leq 3$, and $f \in C\left([0,1] \times \mathbb{R}_{+}^{3}, \mathbb{R}_{+}\right) \quad\left(\mathbb{R}_{+}=[0,+\infty)\right)$. The existence of positive solutions is obtained by the Krasnoselskii-Zabreiko fixed point theorem. And by using the method of lower and upper solutions, the authors discussed the uniqueness of positive solution of this problem.

On the other hand, the fractional differential equations with the $p$-Laplacian operator can describe various phenomena, such as the flow of some specific fluid. Hence, the study of fractional differential equations with the $p$-Laplacian operator is gaining much significance and attention (see $[16,18-21,37-39,48-50])$. For example, in [48], in order to research the mechanics phenomenon of turbulent flow in a porous medium, the author introduced the following type equation:

$$
\left(\phi_{p}\left(x^{\prime}(t)\right)\right)^{\prime}=f\left(t, x(t), x^{\prime}(t)\right),
$$

where $\phi_{p}(p>1)$ is the $p$-Laplacian operator, i.e., $\phi_{p}(s)=$ $|s|^{p-2} s(p>1)$. In the past thirty years, many papers discussed 
equation (2) with different boundary value conditions and then drew related conclusion in practice. We refer the reader to $[49,50]$ and the references cited therein. Recently, Liu et al. in [16] investigated the existence of positive solution of the following fractional differential equations with $p$-Laplacian operator:

$$
\left\{\begin{array}{l}
D_{0+}^{\alpha} \phi_{p}\left({ }^{c} D_{0+}^{\beta} x(t)\right)=f\left(t, x(t),{ }^{c} D_{0+}^{\beta} x(t)\right), \\
{ }^{c} D_{0+}^{\beta} x(0)=x^{\prime}(0)=0, \\
x(1)=r_{1} x(\eta), \quad{ }^{c} D_{0+}^{\beta} x(1)=r_{2}^{c} D_{0+}^{\beta} x(\xi),
\end{array}\right.
$$

where $D_{0+}^{\alpha}$ is the Riemann-Liouville's fractional derivative and ${ }^{c} D_{0+}^{\beta}$ is the Caputo fractional derivative, $1<\alpha, \beta \leq 2, r_{1}$, $r_{1} \geq 0, \quad \phi_{p}(p>1)$ is the $p$-Laplacian operator, and $f \in C\left([0,1] \times \mathbb{R}_{+} \times \mathbb{R}_{-}, \mathbb{R}_{+}\right)\left(\mathbb{R}_{-}=(-\infty, 0]\right)$. The existence of lower and upper solutions is obtained by using the monotone iterative technique.

Based on the above illustration, it is of significance to study the fractional differential equations with $p$-Laplacian operator. In this article, we investigate the following fractional differential equation, which is a generalized form of the problem (2):

$$
\begin{array}{r}
{ }^{c} D_{0+}^{\alpha} \phi_{p}\left(D_{0+}^{\beta} v(t)\right)+f\left(t, v(t), D_{0+}^{\beta_{1}} v(t), \ldots, D_{0+}^{\beta_{n-2}} v(t)\right)=0, \\
0<t<1,
\end{array}
$$

with the boundary conditions

$$
\begin{aligned}
& v(0)= D_{0+}^{v_{i}} v(0)=D_{0+}^{\beta} v(0)=\left(\phi_{p}\left(D_{0+}^{\beta} v(0)\right)\right)^{\prime}=0, \\
& i=1, \ldots, n-2, \\
& D_{0+}^{r_{0}} v(1)= \lambda_{1} \int_{0}^{1} h_{1}(s) D_{0+}^{r_{1}} v(s) d A_{1}(s) \\
&+\lambda_{2} \int_{0}^{\eta} h_{2}(s) D_{0+}^{r_{2}} v(s) d A_{2}(s)+\lambda_{3} \sum_{j=1}^{\infty} \rho_{j} D_{0+}^{r_{3}} v\left(\eta_{j}\right),
\end{aligned}
$$

where ${ }^{c} D_{0+}^{\alpha}$ denotes the Caputo fractional derivative; $D_{0+}^{\beta}$ denotes the Riemann-Liouville's fractional derivative; $\phi_{p}$ is the $p$-Laplacian operator, i.e., $\phi_{p}(s)=|s|^{p-2} s(p>1)$, $1<\alpha \leq 2, \quad n-1<\beta \leq n(n \geq 3), \quad 1<\beta-\beta_{n-2} \leq 2, \quad i-1<\beta_{i}$, $v_{i} \leq i, \quad \beta_{n-2}-v_{i} \leq n-2-i(i=1,2, \ldots, n-2), \quad \beta_{n-2} \leq r_{k} \leq$ $r_{0} \leq \beta-1(k=1,2,3), 0<\eta \leq 1, \quad 0<\eta_{1} \leq \eta_{2} \leq \cdots \leq 1, \rho_{j} \geq 0$ $(j=1,2, \ldots), \lambda_{k} \geq 0(k=1,2,3) ; h_{1}, h_{2}:(0,1) \longrightarrow \mathbb{R}_{+}=[0$, $+\infty)$ are continuous with $h_{1}, h_{2} \in L^{1}(0,1) ; \int_{0}^{1} h_{1}(s) v(s)$ $d A_{1}(s), \int_{0}^{1} h_{2}(s) v(s) d A_{2}(s)$ denote the Riemann-Stieltjes integral, in which $A_{1}, A_{2}:[0,1] \longrightarrow \mathbb{R}$ are functions of bounded variation; and $f:(0,1) \times(0,+\infty)^{n-1} \longrightarrow \mathbb{R}_{+}$is continuous. Furthermore, we also consider the following equation with a parameter:

$$
\begin{array}{r}
{ }^{c} D_{0+}^{\alpha} \phi_{p}\left(D_{0+}^{\beta} v(t)\right)+\lambda f\left(t, v(t), D_{0+}^{\beta_{1}} v(t), \ldots, D_{0+}^{\beta_{n-2}} v(t)\right)=0, \\
0<t<1,
\end{array}
$$

with the boundary conditions (5), where $\lambda>0$ is a parameter. In fact, we regard the problem (6)-(5) as a new problem for modeling the problem (4)-(5) involving a parameter. In this paper, a nonnegative function $v \in C[0,1]$ is called a positive solution of boundary value problem (4)-(5) (resp. (6)-(5)) if it satisfies the problem (4)-(5) (resp. (6)-(5)) for $t \in[0,1]$ and $v(t)>0$ for $t \in(0,1]$.

In this paper, we make the following hypothesis:

$\left(\mathbf{V}_{1}\right) f:(0,1) \times(0,+\infty)^{n-1} \longrightarrow \mathbb{R}_{+}$is continuous and satisfies

$$
\begin{aligned}
f\left(t, u_{1}, \ldots, u_{n-1}\right)= & F\left(t, u_{1}, \ldots, u_{n-1}, u_{1}, \ldots, u_{n-1}\right) \\
& +G\left(t, u_{1}, \ldots, u_{n-1}, u_{1}, \ldots, u_{n-1}\right),
\end{aligned}
$$

for all $\left(t, u_{1}, \ldots, u_{n-1}\right) \in(0,1) \times(0,+\infty)^{n-1}$, where $F, G:(0,1) \times(0,+\infty)^{2(n-1)} \longrightarrow \mathbb{R}_{+}$are continuous.

$\left(\mathbf{V}_{2}\right)$ For any $t \in(0,1),\left(v_{1}, \ldots, v_{n-1}\right) \in(0,+\infty)^{n-1}$, $F\left(t, u_{1}, \ldots, u_{n-1}, v_{1}, \ldots, v_{n-1}\right)$, and $G\left(t, u_{1}, \ldots, u_{n-1}, v_{1}\right.$, $\left.\ldots, v_{n-1}\right)$ are nondecreasing in $\left(u_{1}, \ldots, u_{n-1}\right) \in(0$, $+\infty)^{n-1}$, and for any $t \in(0,1),\left(u_{1}, \ldots, u_{n-1}\right) \in(0$, $+\infty)^{n-1}, F\left(t, u_{1}, \ldots, u_{n-1}, v_{1}, \ldots, v_{n-1}\right)$, and $G\left(t, u_{1}\right.$, $\left.\ldots, u_{n-1}, v_{1}, \ldots, v_{n-1}\right)$ are nonincreasing in $\left(v_{1}, \ldots\right.$, $\left.v_{n-1}\right) \in(0,+\infty)^{n-1}$.

$\left(\mathbf{V}_{3}\right)$ For any $l \in(0,1)$, there exists $\varphi(l) \in\left(l^{q-1}, 1\right]$ such that for any $t \in(0,1),\left(u_{1}, \ldots, u_{n-1}\right)$ and $\left(v_{1}, \ldots\right.$, $\left.v_{n-1}\right) \in(0,+\infty)^{n-1}$ :

$$
\begin{aligned}
& F\left(t, l u_{1}, \ldots, l u_{n-1}, l^{-1} v_{1}, \ldots, l^{-1} v_{n-1}\right) \\
& \quad \geq \varphi^{p-1}(l) F\left(t, u_{1}, \ldots, u_{n-1}, v_{1}, \ldots, v_{n-1}\right), \\
& G\left(t, l u_{1}, \ldots, l u_{n-1}, l^{-1} v_{1}, \ldots, l^{-1} v_{n-1}\right) \\
& \quad \geq l^{p-1} G\left(t, u_{1}, \ldots, u_{n-1}, v_{1}, \ldots, v_{n-1}\right) .
\end{aligned}
$$

$\left(\mathbf{V}_{4}\right)$ There exists $\varrho>0$ such that for any $t \in(0,1)$, $\left(u_{1}, \ldots, u_{n-1}\right)$ and $\left(v_{1}, \ldots, v_{n-1}\right) \in(0,+\infty)^{n-1}$ :

$$
\begin{aligned}
& F\left(t, u_{1}, \ldots, u_{n-1}, v_{1}, \ldots, v_{n-1}\right) \\
& \quad \geq \varrho^{p-1} G\left(t, u_{1}, \ldots, u_{n-1}, v_{1}, \ldots, v_{n-1}\right) .
\end{aligned}
$$

$\left(\mathbf{V}_{5}\right)$

$$
\begin{aligned}
0 & <\int_{0}^{1}(1-\tau)^{\alpha-1} F(\tau, \underbrace{1,1, \ldots, 1}_{n-1 \text { times }}, \underbrace{\tau^{\beta-1}, \tau^{\beta-1}, \ldots, \tau^{\beta-1}}_{n-1 \text { times }}) d \tau \\
& <+\infty \\
0 & <\int_{0}^{1}(1-\tau)^{\alpha-1} G(\tau, \underbrace{1,1, \ldots, 1}_{n-1 \text { times }}, \underbrace{\tau^{\beta-1}, \tau^{\beta-1}, \ldots, \tau^{\beta-1}}_{n-1 \text { times }}) d \tau \\
& <+\infty .
\end{aligned}
$$


Our main results are as follows.

Theorem 1. Suppose that $\left(\mathbf{V}_{1}\right)-\left(\mathbf{V}_{5}\right)$ hold, then the problem (4)-(5) has a unique solution $v^{*} \in P$, and there exists $\delta \in(0,1)$ such that

$$
\frac{\Gamma\left(\beta-\beta_{n-2}\right) \delta}{\Gamma(\beta)} t^{\beta-1} \leq v^{*}(t) \leq \frac{\Gamma\left(\beta-\beta_{n-2}\right)}{\Gamma(\beta) \delta} t^{\beta-1}, \quad t \in[0,1] .
$$

$$
\begin{aligned}
& v_{n}(t)=I_{0^{+}}^{\beta_{n-2}}\left\{\int _ { 0 } ^ { 1 } J ( t , s ) \phi _ { q } \left(\frac { 1 } { \Gamma ( \alpha ) } \int _ { 0 } ^ { s } ( s - \tau ) ^ { \alpha - 1 } F \left(\tau, I_{0^{+}-2}^{\beta_{n-1}}(\tau), I_{0^{+}}^{\beta_{n-2}-\beta_{1}} v_{n-1}(\tau), \ldots, I_{0^{+}}^{\beta_{n-2}-\beta_{n-3}} v_{n-1}(\tau), v_{n-1}(\tau), I_{0^{+}}^{\beta_{n-2}} \widetilde{v}_{n-1}(\tau),\right.\right.\right. \\
& \left.\left.I_{0^{+}}^{\beta_{n-2}-\beta_{1}} \widetilde{v}_{n-1}(\tau), \ldots, I_{0^{+}}^{\beta_{n-2}-\beta_{n-3}} \widetilde{v}_{n-1}(\tau), \widetilde{v}_{n-1}(\tau)\right) d \tau\right) d s \\
& +\int_{0}^{1} J(t, s) \phi_{q}\left(\frac { 1 } { \Gamma ( \alpha ) } \int _ { 0 } ^ { s } ( s - \tau ) ^ { \alpha - 1 } G \left(\tau, I_{0^{+}}^{\beta_{n-2}} v_{n-1}(\tau), I_{0^{+}}^{\beta_{n-2}-\beta_{1}} v_{n-1}(\tau), \ldots, I_{0^{+}}^{\beta_{n-2}-\beta_{n-3}} v_{n-1}(\tau), v_{n-1}(\tau), I_{0^{+}}^{\beta_{n-2}} \widetilde{v}_{n-1}(\tau)\right.\right. \\
& \left.\left.\left.I_{0^{+}}^{\beta_{n-2}-\beta_{1}} \widetilde{v}_{n-1}(\tau), \ldots, I_{0^{+}}^{\beta_{n-2}-\beta_{n-3}} \widetilde{v}_{n-1}(\tau), \widetilde{v}_{n-1}(\tau)\right) d \tau\right) d s\right\} \\
& \widetilde{v}_{n}(t)=I_{0^{+}}^{\beta_{n-2}}\left\{\int _ { 0 } ^ { 1 } J ( t , s ) \phi _ { q } \left(\frac { 1 } { \Gamma ( \alpha ) } \int _ { 0 } ^ { s } ( s - \tau ) ^ { \alpha - 1 } F \left(\tau, I_{0^{+}}^{\beta_{n-2}} \widetilde{v}_{n-1}(\tau), I_{0^{+}}^{\beta_{n-2}-\beta_{1}} \widetilde{v}_{n-1}(\tau), \ldots, I_{0^{+}}^{\beta_{n-2}-\beta_{n-3}} \widetilde{v}_{n-1}(\tau), \widetilde{v}_{n-1}(\tau), I_{0^{+}}^{\beta_{n-2}} v_{n-1}(\tau)\right.\right.\right. \\
& \left.I_{0^{+}}^{\beta_{n-2}-\beta_{1}} v_{n-1}(\tau), \ldots, I_{0^{+}}^{\beta_{n-2}-\beta_{n-3}} v_{n-1}(\tau), v_{n-1}(\tau) d \tau\right) d s \\
& +\int_{0}^{1} J(t, s) \phi_{q}\left(\frac { 1 } { \Gamma ( \alpha ) } \int _ { 0 } ^ { s } ( s - \tau ) ^ { \alpha - 1 } G \left(\tau, I_{0^{+}}^{\beta_{n-2}} \widetilde{v}_{n-1}(\tau), I_{0^{+}}^{\beta_{n-2}-\beta_{1}} \widetilde{v}_{n-1}(\tau), \ldots, I_{0^{+}}^{\beta_{n-2}-\beta_{n-3}} \widetilde{v}_{n-1}(\tau), \widetilde{v}_{n-1}(\tau), I_{0^{+}}^{\beta_{n-2}} v_{n-1}(\tau)\right.\right. \\
& \left.\left.\left.I_{0^{+}}^{\beta_{n-2}-\beta_{1}} v_{n-1}(\tau), \ldots, I_{0^{+}}^{\beta_{n-2}-\beta_{n-3}} v_{n-1}(\tau), v_{n-1}(\tau)\right) d \tau\right) d s\right\}
\end{aligned}
$$

And for any initial values $v_{0}, \widetilde{v}_{0} \in P_{h}$, by structuring the following sequences:$$
n=1,2, \ldots
$$

we have

$$
\begin{aligned}
\sup _{t \in[0,1]}\left|v_{n}(t)-v^{*}(t)\right| & \longrightarrow 0, \\
\sup _{t \in[0,1]}\left|\widetilde{v}_{n}(t)-v^{*}(t)\right| & \longrightarrow 0, \\
\text { as } n & \longrightarrow \infty .
\end{aligned}
$$

Theorem 2. Suppose that $\left(\mathbf{V}_{1}\right)-\left(\mathbf{V}_{5}\right)$ hold, then for any $\lambda>0$, the problem (6)-(5) have a unique solution $v_{\lambda}^{*} \in P$, and there exists $\delta_{\lambda} \in(0,1)$ such that

And moreover,

(1) If there exists $\gamma \in(0,1)$ such that

$$
\varphi(l) \geq \frac{1}{\varrho}\left(l^{\gamma}-l\right)+l^{\gamma}, \quad \forall l \in(0,1),
$$

then $v_{\lambda}^{*}$ is continuous with respect to $\lambda \in(0,+\infty)$. That is, for any $\lambda_{0} \in(0,+\infty)$, 


$$
\sup _{t \in[0,1]}\left|v_{\lambda}^{*}(t)-v_{\lambda_{0}}^{*}(t)\right| \longrightarrow 0, \quad \text { as } \lambda \longrightarrow \lambda_{0} \text {. }
$$

(2) If

$$
\varphi(l) \geq \frac{1}{\varrho}\left(l^{1 / 2}-l\right)+l^{1 / 2}, \quad \forall l \in(0,1),
$$

then $0<\lambda_{1}<\lambda_{2}$ implies $v_{\lambda_{1}}^{*}<v_{\lambda_{2}}^{*}$.

(3) If there exists $\gamma \in(0,1 / 2)$ such that

$$
\varphi(l) \geq \frac{1}{\varrho}\left(l^{\gamma}-l\right)+l^{\gamma}, \quad \forall l \in(0,1),
$$

then

$$
\begin{aligned}
& \lim _{\lambda \longrightarrow 0^{+}} \sup _{t \in[0,1]}\left|v_{\lambda}^{*}(t)\right|=0, \\
& \lim _{\lambda \longrightarrow+\infty} \sup _{t \in[0,1]}\left|v_{\lambda}^{*}(t)\right|=+\infty .
\end{aligned}
$$

The key argument of the problem (4)-(5) and (6)-(5) is the iterative positive solution by applying the method of reducing the order of fractional derivative and the fixed point theory of mixed monotone operator. The method of reducing the order of fractional derivative is based on certain semigroup properties of the Riemann-Liouville's fractional integral and derivative. We refer the reader to $[3,24,25,34,36,46]$. In this paper, different orders of Riemann-Liouville's fractional derivative are involved in the nonlinearity $f$, which is solved in a more complex space, in most cases. By using the method of reduction, we transform the problem (4)-(5) (resp. (6)-(5)) into an equivalent and low-order problem, in which the nonlinearity $f$ contains no fractional derivative. Therefore, the work could proceed in the space $C[0,1]$, which is more interesting and meaningful. On the other hand, by using the properties of relevant Green's function and cone, the theory of mixed monotone operator could be applied on the research of fractional boundary value problems. We suggest that one refers to $[13,17,24,31,32,34,47]$. In this paper, by structuring a suitable mixed monotone operator, the problem (4)-(5) (resp. (6)-(5)), which contains the $p$-Laplacian operator, is solved. In $[15,16]$, the positive solutions are obtained under the assumption that the nonlinear term $f$ is continuous. But, we obtain the uniqueness of positive solution with the assumption that the nonlinear term may be singular in regard to both the time variable and space variables. Compared with [15], our equation contains $p$ Laplacian operator, which is more general. Compared with [16], our boundary conditions are of general significance, which would be used to describe more phenomena in practice.

The paper is organized as follows. In Section 2, we present some relevant definitions and lemmas. And we transform the problem (4)-(5) (resp. (6)-(5)) into an equivalent and low-order problem (28)-(29) (resp. (44)(29)) and obtain some properties of the corresponding Green's function. In Section 3, we give the proof of our main results, as one researches the uniqueness of iterative positive solutions for the problem (4)-(5) and the other one analyses the dependence of solution upon parameter for the problem (6)-(5). In Section 4, we give two examples to illustrate our theoretical results. In Section 5, we give a conclusion of our main results.

\section{Preliminaries and Lemmas}

Definition 1. Let $\alpha>0$. The Riemann-Liouville fractional integral of order $\alpha$ of a function $v:(0, \infty) \longrightarrow \mathbb{R}$ is given by

$$
I_{0^{+}}^{\alpha} v(t)=\frac{1}{\Gamma(\alpha)} \int_{0}^{t}(t-s)^{\alpha-1} v(s) d s,
$$

provided that the right-hand side is pointwise defined on $(0, \infty)$.

Definition 2. Let $\alpha>0$. The Riemann-Liouville fractional derivative of order $a$ of a continuous function $v$ : $(0, \infty) \longrightarrow \mathbb{R}$ is given by

$$
\begin{aligned}
D_{0^{+}}^{\alpha} v(t)= & \left(\frac{d}{d t}\right)^{n} I_{0^{+}}^{n-\alpha} v(t)=\frac{1}{\Gamma(n-\alpha)}\left(\frac{d}{d t}\right)^{n} \\
& \cdot \int_{0}^{t} \frac{v(s)}{(t-s)^{\alpha-n+1}} d s,
\end{aligned}
$$

where $n=[\alpha]+1,[\alpha]$ denotes the integer part of the number $\alpha$, provided that the right-hand side is pointwise defined on $(0, \infty)$. In particular, if $\alpha=n \in \mathbb{N}_{+}$, then $D_{0^{+}}^{\alpha} v(t)=v^{(n)}(t)$.

Definition 3. Let $\alpha>0$. The Caputo fractional derivative of order $\alpha$ of a continuous function $v:(0, \infty) \longrightarrow \mathbb{R}$ is given by

$$
{ }^{c} D_{0^{+}}^{\alpha} v(t)=\frac{1}{\Gamma(n-\alpha)} \int_{0}^{t} \frac{v^{(n)}(s)}{(t-s)^{\alpha-n+1}} d s,
$$

where $n=[\alpha]+1,[\alpha]$ denotes the integer part of the number $\alpha$, provided that the right-hand side is pointwise defined on $(0, \infty)$. In particular, if $\alpha=n \in \mathbb{N}_{+}$, then ${ }^{c} D_{0^{+}}^{\alpha} v(t)=v^{(n)}(t)$.

Lemma 1 (see [7]). Let $\alpha>0$. If we assume $v \in C(0,1) \cap$ $L^{1}(0,1)$, then the equation

$$
D_{0^{+}}^{\alpha} v(t)=0
$$

has

$$
v(t)=c_{1} t^{\alpha-1}+c_{2} t^{\alpha-2}+\cdots+c_{n} t^{\alpha-n}, \quad c_{i} \in \mathbb{R}, i=1,2, \ldots, n,
$$


as the unique solution, where $n$ is the smallest integer greater than or equal to $\alpha$.

Lemma 2 (see [7]). Let $v \in C(0,1) \cap L^{1}(0,1)$ and $D_{0^{+}}^{\alpha} v \in$ $C(0,1) \cap L^{1}(0,1)$. Then,

$$
\begin{array}{r}
I_{0^{+}}^{\alpha} D_{0^{+}}^{\alpha} v(t)=v(t)+c_{1} t^{\alpha-1}+c_{2} t^{\alpha-2}+\cdots+c_{n} t^{\alpha-n}, \\
c_{i} \in \mathbb{R}, i=1,2, \ldots, n,
\end{array}
$$

where $n$ is the smallest integer greater than or equal to $\alpha$.

Lemma 3 (see [4]). Let $v \in A C^{n}[0,1]$. Then,

$$
\begin{array}{r}
I_{0^{+}}^{\alpha c} D_{0^{+}}^{\alpha} v(t)=v(t)+c_{0}+c_{1} t+\cdots+c_{n-1} t^{n-1}, \\
c_{i} \in \mathbb{R}, i=1,2, \ldots, n,
\end{array}
$$

where $n=[\alpha]+1$ for $\alpha \notin \mathbb{N}_{+}$and $n=\alpha$ for $\alpha \in \mathbb{N}_{+}$.

Lemma 4 (see [4]).

(1) If $u \in L^{1}(0,1), \alpha \geq \beta>0$, then

$$
\begin{aligned}
& D_{0^{+}}^{\alpha} I_{0^{+}}^{\alpha} u(t)=u(t), \\
& D_{0^{+}}^{\beta} I_{0^{+}}^{\alpha} u(t)=I_{0^{+}}^{\alpha-\beta} u(t) .
\end{aligned}
$$

(2) Let $\alpha \in(n-1, n)$. If $u(0)=u^{\prime}(0)=\cdots=u^{(n-1)}(0)$, then ${ }^{c} D_{0^{+}}^{\alpha} v(t)=D_{0^{+}}^{\alpha} v(t)$.

Let $u=D_{0^{+}}^{\beta_{n-2}} v$, then the problem (4)-(5) could be transformed to the following fractional differential equation:

$$
\begin{aligned}
& { }^{c} D_{0+}^{\alpha} \phi_{p}\left(D_{0+}^{\beta-\beta_{n-2}} u(t)\right)+f\left(t, I_{0+}^{\beta_{n-2}} u(t),\right. \\
& \left.I_{0+}^{\beta_{n-2}-\beta_{1}} u(t), \ldots, I_{0+}^{\beta_{n-2}-\beta_{n-3}} u(t), u(t)\right)=0, \quad 0<t<1,
\end{aligned}
$$

with the boundary conditions

$$
\begin{aligned}
D_{0+}^{v_{n-2}-\beta_{n-2}} u(0)= & D_{0+}^{\beta-\beta_{n-2}} u(0)=\left(\phi_{p}\left(D_{0+}^{\beta-\beta_{n-2}} u(0)\right)\right)^{\prime}=0, \\
D_{0+}^{r_{0}-\beta_{n-2}} u(1)= & \lambda_{1} \int_{0}^{1} h_{1}(s) D_{0+}^{r_{1}-\beta_{n-2}} u(s) d A_{1}(s) \\
& +\lambda_{2} \int_{0}^{\eta} h_{2}(s) D_{0+}^{r_{2}-\beta_{n-2}} u(s) d A_{2}(s) \\
& +\lambda_{3} \sum_{j=1}^{\infty} \rho_{j} D_{0+}^{r_{3}-\beta_{n-2}} u\left(\eta_{j}\right),
\end{aligned}
$$

where $1<\beta-\beta_{n-2} \leq 2$.

Lemma 5. If the problem (4)-(5) have a solution $v \in C[0,1]$, then $u=D_{0^{+}}^{\beta_{n-2}} v$ is a solution of the problem (28)-(29). And in turn, if the problem (28)-(29) have a solution $u \in C[0,1]$, then $v=I_{0^{+}}^{\beta_{n-2}} u$ is a solution of the problem (4)-(5).

Proof. Let

$$
u(t)=D_{0^{+}}^{\beta_{n-2}} v(t), \quad t \in[0,1],
$$

where $v \in C[0,1]$ is a solution of the problem (4)-(5). By using Lemma 2, we have

$$
v(t)=I_{0^{+}}^{\beta_{n-2}} u(t)-c_{1} t^{\beta_{n-2}-1}-\cdots-c_{n-2} t^{\beta_{n-2}-(n-2)},
$$

where $c_{i} \in \mathbb{R}(i=1,2, \ldots, n-2)$. By the boundary condition $v(0)=0$, we get $c_{n-2}=0$. So, we have

$$
v(t)=I_{0^{+}}^{\beta_{n-2}} u(t)-c_{1} t^{\beta_{n-2}-1}-\cdots-c_{n-3} t^{\beta_{n-2}-(n-3)} .
$$

Furthermore, we obtain

$$
\begin{aligned}
D_{0^{+}}^{v_{1}} v(t)= & I_{0^{+}}^{\beta_{n-2}-v_{1}} u(t)-c_{1} \frac{\Gamma\left(\beta_{n-2}\right)}{\Gamma\left(\beta_{n-2}-v_{1}\right)} t^{\beta_{n-2}-v_{1}-1} \\
& -c_{2} \frac{\Gamma\left(\beta_{n-2}-1\right)}{\Gamma\left(\beta_{n-2}-v_{1}-1\right)} t^{\beta_{n-2}-v_{1}-2}-\cdots-c_{n-3} \\
& \cdot \frac{\Gamma\left(\beta_{n-2}-(n-4)\right)}{\Gamma\left(\beta_{n-2}-v_{1}-(n-4)\right)} t^{\beta_{n-2}-v_{1}-(n-3)},
\end{aligned}
$$

which combines with the boundary condition $D_{0+}^{\nu_{1}} v(0)=0$ meaning $c_{n-3}=0$. In a similar manner, we obtain $c_{n-4}=$ $\cdots=c_{1}=0$ based on the boundary condition $D_{0+}^{v_{2}}$ $v(0)=\cdots=D_{0+}^{v_{n-3}} v(0)=0$. Thus, we have

$$
v(t)=I_{0^{+}}^{\beta_{n-2}} u(t), \quad t \in[0,1] .
$$

Applying the operator $D_{0^{+}}^{\beta}$ to both sides of this equality, we have

$$
\begin{aligned}
D_{0^{+}}^{\beta} v(t) & =D_{0^{+}}^{\beta}\left(I_{0^{+}}^{\beta_{n-2}} u(t)\right)=\frac{d^{n}}{d t^{n}} I_{0^{+}}^{n-\beta}\left(I_{0^{+}}^{\beta_{n-2}} u(t)\right) \\
& =\frac{d^{n}}{d t^{n}} I_{0^{+}}^{n-\left(\beta-\beta_{n-2}\right)} u(t) \\
& =\frac{d^{2}}{d t^{2}}\left(\frac{d^{n-2}}{d t^{n-2}} I_{0^{+}}^{n-2}\right) I_{0^{+}}^{2-\left(\beta-\beta_{n-2}\right)} u(t) \\
& =\frac{d^{2}}{d t^{2}} I_{0^{+}}^{2-\left(\beta-\beta_{n-2}\right)} u(t) \\
& =D_{0^{+}}^{\beta-\beta_{n-2}} u(t) .
\end{aligned}
$$


Similarly, we know

$D_{0+}^{v_{n-2}} v(t)=D_{0+}^{v_{n-2}-\beta_{n-2}} u(t)$

$D_{0^{+}}^{r_{i}} v(t)=D_{0^{+}}^{r_{i}} I_{0^{+}}^{\beta_{n-2}} u(t)=D_{0^{+}}^{r_{i}-\beta_{n-2}} u(t), \quad i=0,1,2,3$.

In addition, by means of Lemma 4, we can obtain

$D_{0^{+}}^{\beta_{i}} v(t)=D_{0^{+}}^{\beta_{i}} I_{0^{+}}^{\beta_{n-2}} u(t)=I_{0^{+}}^{\beta_{n-2}-\beta_{i}} u(t), \quad i=1,2, \ldots, n-3$.

According to (30), (34), (36), and (38), we have

$$
\begin{aligned}
& { }^{c} D_{0+}^{\alpha} \phi_{p}\left(D_{0+}^{\beta-\beta_{n-2}} u(t)\right)+f\left(t, I_{0+}^{\beta_{n-2}} u(t), I_{0+}^{\beta_{n-2}-\beta_{1}}\right. \\
& \left.\left.\quad \cdot u(t), \ldots, I_{0+}^{\beta_{n-2}-\beta_{n-3}} u(t), u(t)\right)\right) \\
& \quad={ }^{c} D_{0+}^{\alpha} \phi_{p}\left(D_{0+}^{\beta} v(t)\right)+f\left(t, v(t), D_{0+}^{\beta_{1}} v(t), \ldots, D_{0+}^{\beta_{n-2}} v(t)\right) \\
& \quad=0 .
\end{aligned}
$$

It follows from (35)-(37) that

$$
\begin{aligned}
& D_{0+}^{\beta-\beta_{n-2}} u(0)=D_{0+}^{\beta} v(0)=0, \\
& \left(\phi_{p}\left(D_{0+}^{\beta-\beta_{n-2}} u(0)\right)\right)^{\prime}=\left(\phi_{p}\left(D_{0+}^{\beta} v(0)\right)\right)^{\prime}=0, \\
& D_{0+}^{\nu_{n-2}-\beta_{n-2}} u(0)=D_{0+}^{v_{n-2}} v(0)=0 \\
& D_{0+}^{r_{0}-\beta_{n-2}} u(1)=D_{0+}^{r_{0}} v(1)=\lambda_{1} \int_{0}^{1} h_{1}(s) D_{0+}^{r_{1}} v(s) d A_{1}(s) \\
& +\lambda_{2} \int_{0}^{\eta} h_{2}(s) D_{0+}^{r_{2}} v(s) d A_{2}(s) \\
& +\lambda_{3} \sum_{i=1}^{\infty} \rho_{i} D_{0+}^{r_{3}} v\left(\eta_{i}\right) \\
& =\lambda_{1} \int_{0}^{1} h_{1}(s) D_{0+}^{r_{1}-\beta_{n-2}} u(s) d A_{1}(s) \\
& +\lambda_{2} \int_{0}^{\eta} h_{2}(s) D_{0+}^{r_{2}-\beta_{n-2}} u(s) d A_{2}(s) \\
& +\lambda_{3} \sum_{i=1}^{\infty} \rho_{i} D_{0+}^{r_{3}-\beta_{n-2}} u\left(\eta_{i}\right)
\end{aligned}
$$

On the basis of (39)-(43), we know $u=D_{0^{+}}^{\beta_{n-2}} v$ is a solution of the problem (28)-(29).

On the other hand, if the problem (28)-(29) has a solution $u \in C[0,1]$, then $v=I_{0^{+}}^{\beta_{n-2}} u$ is a solution of the problem (4)-(5). Since the proof is similar to Lemma 3 in [44], we omit it here.

Remark 5. According to Lemma 5, we conclude that the work on considering the solution of the problem (4)-(5) is equivalent to the search for the solution of (28)-(29). Similarly, we can prove the work on the problem (6)-(5) is equivalent to the following equation:

$$
\begin{gathered}
{ }^{c} D_{0+}^{\alpha} \phi_{p}\left(D_{0+}^{\beta-\beta_{n-2}} u(t)\right)+\lambda f\left(t, I_{0+}^{\beta_{n-2}} u(t), I_{0+}^{\beta_{n-2}-\beta_{1}}\right. \\
\cdot u(t), \ldots, I_{0+}^{\beta_{n-2}-\beta_{n-3}} u(t), u(t)=0, \quad 0<t<1,
\end{gathered}
$$

with the boundary conditions (29).

Lemma 6. Let $x \in C(0,1) \cap L^{1}(0,1)$. Then, the fractional differential equation

$$
D_{0+}^{\beta-\beta_{n-2}} u(t)+x(t)=0, \quad 0<t<1,1<\beta-\beta_{n-2} \leq 2,
$$

with the boundary conditions

$$
\begin{aligned}
D_{0+}^{v_{n-2}-\beta_{n-2}} u(0)= & 0 \\
D_{0+}^{r_{0}-\beta_{n-2}} u(1)= & \lambda_{1} \int_{0}^{1} h_{1}(s) D_{0+}^{r_{1}-\beta_{n-2}} u(s) d A_{1}(s) \\
& +\lambda_{2} \int_{0}^{\eta} h_{2}(s) D_{0+}^{r_{2}-\beta_{n-2}} u(s) d A_{2}(s) \\
& +\lambda_{3} \sum_{j=1}^{\infty} \rho_{j} D_{0+}^{r_{3}-\beta_{n-2}} u\left(\eta_{j}\right)
\end{aligned}
$$

has a unique solution

$$
u(t)=\int_{0}^{1} J(t, s) x(s) d s,
$$

where

$$
\begin{aligned}
J(t, s)= & J_{0}(t, s)+t^{\beta-\beta_{n-2}-1}\left(\int_{0}^{1} J_{1}(\tau, s) h_{1}(\tau) d A_{1}(\tau)\right) \\
& +t^{\beta-\beta_{n-2}-1}\left(\int_{0}^{\eta} J_{2}(\tau, s) h_{2}(\tau) d A_{2}(\tau)\right) \\
& +t^{\beta-\beta_{n-2}-1} \sum_{j=1}^{\infty} \rho_{j} J_{3}\left(\eta_{j}, s\right)
\end{aligned}
$$

in which 


$$
\begin{aligned}
& J_{0}(t, s)=\frac{1}{\Gamma\left(\beta-\beta_{n-2}\right)} \begin{cases}t^{\beta-\beta_{n-2}-1}(1-s)^{\beta-r_{0}-1}-(t-s)^{\beta-\beta_{n-2}-1}, & s \leq t, \\
t^{\beta-\beta_{n-2}-1}(1-s)^{\beta-r_{0}-1}, & t \leq s,\end{cases} \\
& J_{1}(t, s)=\frac{\lambda_{1}}{\sigma \Gamma\left(\beta-\beta_{n-2}\right) \Gamma\left(\beta-r_{1}\right)} \begin{cases}t^{\beta-r_{1}-1}(1-s)^{\beta-r_{0}-1}-(t-s)^{\beta-r_{1}-1}, & s \leq t, \\
t^{\beta-r_{1}-1}(1-s)^{\beta-r_{0}-1}, & t \leq s,\end{cases} \\
& J_{2}(t, s)=\frac{\lambda_{2}}{\sigma \Gamma\left(\beta-\beta_{n-2}\right) \Gamma\left(\beta-r_{2}\right)} \begin{cases}t^{\beta-r_{2}-1}(1-s)^{\beta-r_{0}-1}-(t-s)^{\beta-r_{2}-1}, & s \leq t, \\
t^{\beta-r_{2}-1}(1-s)^{\beta-r_{0}-1}, & t \leq s,\end{cases} \\
& J_{3}(t, s)=\frac{\lambda_{3}}{\sigma \Gamma\left(\beta-\beta_{n-2}\right) \Gamma\left(\beta-r_{3}\right)} \begin{cases}t^{\beta-r_{3}-1}(1-s)^{\beta-r_{0}-1}-(t-s)^{\beta-r_{3}-1}, & s \leq t, \\
t^{\beta-r_{3}-1}(1-s)^{\beta-r_{0}-1}, & t \leq s,\end{cases} \\
& \sigma=\frac{1}{\Gamma\left(\beta-r_{0}\right)}-\frac{\lambda_{1}}{\Gamma\left(\beta-r_{1}\right)} \int_{0}^{1} s^{\beta-r_{1}-1} h_{1}(s) d A_{1}(s)-\frac{\lambda_{2}}{\Gamma\left(\beta-r_{2}\right)} \int_{0}^{\eta} s^{\beta-r_{2}-1} h_{2}(s) d A_{2}(s)-\frac{\lambda_{3}}{\Gamma\left(\beta-r_{3}\right)} \sum_{j=1}^{\infty} \rho_{j} \eta_{j}^{\beta-r_{3}-1} \neq 0 .
\end{aligned}
$$

Obviously, $J:[0,1] \times[0,1] \longrightarrow \mathbb{R}_{+}$is continuous.

Proof. We may apply Lemma 2 to reduce equation (45) to an equivalent integral equation:

$$
u(t)=c_{1} t^{\beta-\beta_{n-2}-1}+c_{2} t^{\beta-\beta_{n-2}-2}-I_{0+}^{\beta-\beta_{n-2}} x(t),
$$

where $c_{1}, c_{2} \in \mathbb{R}$. Since $D_{0+}^{v_{n-2}-\beta_{n-2}} u(0)=0$, we have $c_{2}=0$. So

$$
u(t)=c_{1} t^{\beta-\beta_{n-2}-1}-I_{0+}^{\beta-\beta_{n-2}} x(t) .
$$

Applying the operator $D_{0+}^{r_{i}-\beta_{n-2}}(i=0,1,2,3)$ to both sides of (51), we have

$$
D_{0+}^{r_{i}-\beta_{n-2}} u(t)=c_{1} \frac{\Gamma\left(\beta-\beta_{n-2}\right)}{\Gamma\left(\beta-r_{i}\right)} t^{\beta-r_{i}-1}-I_{0+}^{\beta-r_{i}} x(t), \quad i=0,1,2,3 .
$$

Hence, according to (52) and the boundary condition

$$
\begin{aligned}
D_{0+}^{r_{0}-\beta_{n-2}} u(1)= & \lambda_{1} \int_{0}^{1} h_{1}(s) D_{0+}^{r_{1}-\beta_{n-2}} u(s) d A_{1}(s) \\
& +\lambda_{2} \int_{0}^{\eta} h_{2}(s) D_{0+}^{r_{2}-\beta_{n-2}} u(s) d A_{2}(s) \\
& +\lambda_{3} \sum_{j=1}^{\infty} \rho_{j} D_{0+}^{r_{3}-\beta_{n-2}} u\left(\eta_{j}\right),
\end{aligned}
$$

we can obtain

$$
\begin{aligned}
c_{1}= & \frac{1}{\sigma \Gamma\left(\beta-\beta_{n-2}\right)}\left\{\frac{1}{\Gamma\left(\beta-r_{0}\right)} \int_{0}^{1}(1-s)^{\beta-r_{0}-1} x(s) d s-\frac{\lambda_{1}}{\Gamma\left(\beta-r_{1}\right)}\right. \\
& \cdot \int_{0}^{1}\left(\int_{0}^{s}(s-\tau)^{\beta-r_{1}-1} x(\tau) d \tau\right) h_{1}(s) d A_{1}(s)-\frac{\lambda_{2}}{\Gamma\left(\beta-r_{2}\right)} \\
& \cdot \int_{0}^{\eta}\left(\int_{0}^{s}(s-\tau)^{\beta-r_{2}-1} x(\tau) d \tau\right) h_{2}(s) d A_{2}(s) \\
& \left.-\frac{\lambda_{3}}{\Gamma\left(\beta-r_{3}\right)} \sum_{j=1}^{\infty} \rho_{j} \int_{0}^{\eta_{j}}\left(\eta_{j}-s\right)^{\beta-r_{3}-1} x(s) d s\right\},
\end{aligned}
$$

where

$$
\begin{aligned}
\sigma= & \frac{1}{\Gamma\left(\beta-r_{0}\right)}-\frac{\lambda_{1}}{\Gamma\left(\beta-r_{1}\right)} \int_{0}^{1} s^{\beta-r_{1}-1} h_{1}(s) \\
& -\frac{\lambda_{2}}{\Gamma\left(\beta-r_{2}\right)} \int_{0}^{\eta} s^{\beta-r_{2}-1} h_{2}(s) d A_{2}(s) \\
& -\frac{\lambda_{3}}{\Gamma\left(\beta-r_{3}\right)} \sum_{j=1}^{\infty} \rho_{j} \eta_{j}^{\beta-r_{3}-1} \neq 0 .
\end{aligned}
$$

Then 


$$
\begin{aligned}
& u(t)=-\frac{1}{\Gamma\left(\beta-\beta_{n-2}\right)} \int_{0}^{t}(t-s)^{\beta-\beta_{n-2}-1} x(s) d s+\frac{t^{\beta-\beta_{n-2}-1}}{\sigma \Gamma\left(\beta-\beta_{n-2}\right)}\left\{\frac{1}{\Gamma\left(\beta-r_{0}\right)} \int_{0}^{1}(1-s)^{\beta-r_{0}-1} x(s) d s\right. \\
& -\frac{\lambda_{1}}{\Gamma\left(\beta-r_{1}\right)} \int_{0}^{1}\left(\int_{0}^{s}(s-\tau)^{\beta-r_{1}-1} x(\tau) d \tau\right) h_{1}(s) d A_{1}(s)-\frac{\lambda_{2}}{\Gamma\left(\beta-r_{2}\right)} \\
& \left.\cdot \int_{0}^{\eta}\left(\int_{0}^{s}(s-\tau)^{\beta-r_{2}-1} x(\tau) d \tau\right) h_{2}(s) d A_{2}(s)-\frac{\lambda_{3}}{\Gamma\left(\beta-r_{3}\right)} \sum_{j=1}^{\infty} \rho_{j} \int_{0}^{\eta_{j}}\left(\eta_{j}-s\right)^{\beta-r_{3}-1} x(s) d s\right\} \\
& =-\frac{1}{\Gamma\left(\beta-\beta_{n-2}\right)} \int_{0}^{t}(t-s)^{\beta-\beta_{n-2}-1} x(s) d s \\
& +\frac{1}{\Gamma\left(\beta-\beta_{n-2}\right)} \int_{0}^{1} t^{\beta-\beta_{n-2}-1}(1-s)^{\beta-r_{0}-1} x(s) d s+\frac{t^{\beta-\beta_{n-2}-1}}{\sigma \Gamma\left(\beta-\beta_{n-2}\right)} \int_{0}^{1}(1-s)^{\beta-r_{0}-1} x(s) d s \\
& \times\left\{\frac{\lambda_{1}}{\Gamma\left(\beta-r_{1}\right)} \int_{0}^{1} s^{\beta-r_{1}-1} h_{1}(s) d A_{1}(s)+\frac{\lambda_{2}}{\Gamma\left(\beta-r_{2}\right)} \int_{0}^{\eta} s^{\beta-r_{2}-1} h_{2}(s) d A_{2}(s)+\frac{\lambda_{3}}{\Gamma\left(\beta-r_{3}\right)} \sum_{j=1}^{\infty} \rho_{j} \eta_{j}^{\beta-r_{3}-1}\right\} \\
& -\left\{\frac{\lambda_{1}}{\Gamma\left(\beta-r_{1}\right)} \int_{0}^{1}\left(\int_{0}^{s}(s-\tau)^{\beta-r_{1}-1} x(\tau) d \tau\right) h_{1}(s) d A_{1}(s)+\frac{\lambda_{2}}{\Gamma\left(\beta-r_{2}\right)} \int_{0}^{\eta}\left(\int_{0}^{s}(s-\tau)^{\beta-r_{2}-1} x(\tau) d \tau\right) h_{2}(s) d A_{2}(s)\right. \\
& \left.+\frac{\lambda_{3}}{\Gamma\left(\beta-r_{3}\right)} \sum_{j=1}^{\infty} \rho_{j} \int_{0}^{\eta_{j}}\left(\eta_{j}-s\right)^{\beta-r_{3}-1} x(s) d s\right\} \frac{t^{\beta-\beta_{n-2}-1}}{\sigma \Gamma\left(\beta-\beta_{n-2}\right)} \\
& =\frac{1}{\Gamma\left(\beta-\beta_{n-2}\right)}\left\{\int_{0}^{1} t^{\beta-\beta_{n-2}-1}(1-s)^{\beta-r_{0}-1} x(s) d s-\int_{0}^{t}(t-s)^{\beta-\beta_{n-2}-1} x(s) d s\right\} \\
& +\frac{\lambda_{1} t^{\beta-\beta_{n-2}-1}}{\sigma \Gamma\left(\beta-\beta_{n-2}\right) \Gamma\left(\beta-r_{1}\right)}\left\{\int_{0}^{1}\left(\int_{0}^{1} s^{\beta-r_{1}-1}(1-\tau)^{\beta-r_{0}-1} x(\tau) d \tau-\int_{0}^{s}(s-\tau)^{\beta-r_{1}-1} x(\tau) d \tau\right) h_{1}(s) d A_{1}(s)\right\} \\
& +\frac{\lambda_{2} t^{\beta-\beta_{n-2}-1}}{\sigma \Gamma\left(\beta-\beta_{n-2}\right) \Gamma\left(\beta-r_{2}\right)}\left\{\int_{0}^{\eta}\left(\int_{0}^{1} s^{\beta-r_{2}-1}(1-\tau)^{\beta-r_{0}-1} x(\tau) d \tau-\int_{0}^{s}(s-\tau)^{\beta-r_{2}-1} x(\tau) d \tau\right) h_{2}(s) d A_{2}(s)\right\} \\
& +\frac{\lambda_{3} t^{\beta-\beta_{n-2}-1}}{\sigma \Gamma\left(\beta-\beta_{n-2}\right) \Gamma\left(\beta-r_{3}\right)} \sum_{j=1}^{\infty} \rho_{j}\left(\int_{0}^{1} \eta_{j}^{\beta-r_{3}-1}(1-s)^{\beta-r_{0}-1} x(s) d s-\int_{0}^{\eta_{j}}\left(\eta_{j}-s\right)^{\beta-r_{3}-1} x(s) d s\right) \\
& =\int_{0}^{1} J_{0}(t, s) x(s) d s+\int_{0}^{1} t^{\beta-\beta_{n-2}-1}\left(\int_{0}^{1} J_{1}(s, \tau) x(\tau) d \tau\right) h_{1}(s) d A_{1}(s)+\int_{0}^{\eta} t^{\beta-\beta_{n-2}-1}\left(\int_{0}^{1} J_{2}(s, \tau) x(\tau) d \tau\right) h_{2}(s) d A_{2}(s) \\
& +t^{\beta-\beta_{n-2}-1} \int_{0}^{1} \sum_{j=1}^{\infty} \rho_{j} J_{3}\left(\eta_{j}, s\right) x(s) d s \\
& =\int_{0}^{1} J_{0}(t, s) x(s) d s+\int_{0}^{1} t^{\beta-\beta_{n-2}-1}\left(\int_{0}^{1} J_{1}(\tau, s) h_{1}(\tau) d A_{1}(\tau)\right) x(s) d s \\
& +\int_{0}^{1} t^{\beta-\beta_{n-2}-1}\left(\int_{0}^{\eta} J_{2}(\tau, s) h_{2}(\tau) d A_{2}(\tau)\right) x(s) d s+\int_{0}^{1} t^{\beta-\beta_{n-2}-1} \sum_{j=1}^{\infty} \rho_{j} J_{3}(\eta, s) x(s) d s \\
& =\int_{0}^{1} J(t, s) x(s) d s .
\end{aligned}
$$

The proof is complete. 
Lemma 7. Let $x \in C(0,1) \cap L^{1}(0,1)$ be a given function. Then, the following equation

$$
{ }^{c} D_{0+}^{\alpha} \phi_{p}\left(D_{0+}^{\beta-\beta_{n-2}} u(t)\right)+x(t)=0, \quad 0<t<1,
$$

with the boundary conditions

$$
\begin{aligned}
D_{0+}^{v_{n-2}-\beta_{n-2}} u(0)= & D_{0+}^{\beta-\beta_{n-2}} u(0)=\left(\phi_{p}\left(D_{0+}^{\beta-\beta_{n-2}} u(0)\right)\right)^{\prime}=0, \\
D_{0+}^{r_{0}-\beta_{n-2}} u(1)= & \lambda_{1} \int_{0}^{1} h_{1}(s) D_{0+}^{r_{1}-\beta_{n-2}} u(s) d A_{1}(s) \\
& +\lambda_{2} \int_{0}^{\eta} h_{2}(s) D_{0+}^{r_{2}-\beta_{n-2}} u(s) d A_{2}(s) \\
& +\lambda_{3} \sum_{j=1}^{\infty} \rho_{j} D_{0+}^{r_{3}-\beta_{n-2}} u\left(\eta_{j}\right),
\end{aligned}
$$

has a unique solution

$$
u(t)=\int_{0}^{1} J(t, s) \phi_{q}\left(\int_{0}^{1} H(s, \tau) x(\tau) d \tau\right) d s,
$$

where $\phi_{q}=\phi_{p}^{-1}, J(t, s)$ is defined in Lemma 6, and

$$
H(t, s)=\frac{1}{\Gamma(\alpha)} \begin{cases}(t-s)^{\alpha-1}, & 0 \leq s \leq t \leq 1, \\ 0, & 0 \leq t \leq s \leq 1 .\end{cases}
$$

Proof. Let $\phi_{p}\left(D_{0+}^{\beta-\beta_{n-2}} u(t)\right)=w(t)$. Then, we can strip down the problem (57)-(58) to the following two problems:

$$
\begin{aligned}
& \left\{\begin{array}{l}
{ }^{c} D_{0+}^{\alpha} w(t)+x(t)=0, \quad 0<t<1 \\
w(0)=w^{\prime}(0)=0
\end{array}\right. \\
& \left\{\begin{array}{l}
D_{0+}^{\beta-\beta_{n-2}} u(t)=\phi_{q} w(t), \quad 0<t<1 \\
D_{0+}^{v_{n-2}-\beta_{n-2}} u(0)=0 \\
D_{0+}^{r_{0}-\beta_{n-2}} u(1)=\lambda_{1} \int_{0}^{1} h_{1}(s) D_{0+}^{r_{1}-\beta_{n-2}} u(s) d A_{1}(s) \\
\quad+\lambda_{2} \int_{0}^{\eta} h_{2}(s) D_{0+}^{r_{2}-\beta_{n-2}} u(s) d A_{2}(s)+\lambda_{3} \sum_{j=1}^{\infty} \rho_{j} D_{0+}^{r_{3}-\beta_{n-2}} u\left(\eta_{j}\right)
\end{array}\right.
\end{aligned}
$$

Obviously, the problem (61) can be written as

$$
w(t)=c_{0}+c_{1} t-I_{0+}^{\alpha} x(t) .
$$

The boundary conditions $w(0)=w^{\prime}(0)=0$ imply that $c_{0}=c_{1}=0$. Thus,

$$
w(t)=-I_{0+}^{\alpha} x(t)=-\int_{0}^{1} H(t, s) x(s) d s .
$$

On the other hand, by Lemma 6, we know the problem (62) has a unique solution:

$$
u(t)=-\int_{0}^{1} J(t, s) \phi_{q}(w(s)) d s .
$$

Therefore, the problem (57)-(58) has a unique solution:

$$
u(t)=\int_{0}^{1} J(t, s) \phi_{q}\left(\int_{0}^{1} H(s, \tau) x(\tau) d \tau\right) d s
$$

Lemma 8. Let $\sigma>0$ (defined in Lemma 6$), \lambda_{i} \geq 0(i=1,2,3)$, $\int_{0}^{1} s^{\beta-r_{1}-1} h_{1}(s) d A_{1}(s) \geq 0, \quad \int_{0}^{\eta} s^{\beta-r_{2}-1} h_{2}(s) d A_{2}(s) \geq 0, \quad$ and $0 \leq \sum_{j=1}^{\infty} \rho_{j} \eta_{j}^{\beta-r_{3}-1}<+\infty$. Then, the following properties hold:

(1) $t^{\beta-\beta_{n-2}-1} g_{0}(s) \leq J_{0}(t, s) \leq \Lambda_{0} t^{\beta-\beta_{n-2}-1}$, where $\Lambda_{0}=1$ / $\left(\Gamma\left(\beta-\beta_{n-2}\right)\right)$,

$$
g_{0}(s)=\frac{1}{\Gamma\left(\beta-\beta_{n-2}\right)}(1-s)^{\beta-r_{0}-1}\left(1-(1-s)^{r_{0}-\beta_{n-2}}\right) .
$$

(2) $t^{\beta-r_{i}-1} g_{i}(s) \leq J_{i}(t, s) \leq \Lambda_{i} t^{\beta-r_{i}-1}(i=1,2,3)$, where

$$
\begin{aligned}
g_{i}(s) & =\frac{1}{\Gamma\left(\beta-r_{i}\right)}(1-s)^{\beta-r_{0}-1}\left(1-(1-s)^{r_{0}-r_{i}}\right), \quad i=1,2,3, \\
\Lambda_{i} & =\frac{\lambda_{i}}{\sigma \Gamma\left(\beta-\beta_{n-2}\right) \Gamma\left(\beta-r_{i}\right)}, \quad i=1,2,3 .
\end{aligned}
$$

(3) $h(t) g(s) \leq J(t, s) \leq \Lambda h(t)$, where $h(t)=t^{\beta-\beta_{n-2}-1}$,

$$
\begin{aligned}
g(s)= & g_{0}(s)+\int_{0}^{1} J_{1}(\tau, s) h_{1}(\tau) d A_{1}(\tau) \\
& +\int_{0}^{\eta} J_{2}(\tau, s) h_{2}(\tau) d A_{2}(\tau)+\sum_{j=1}^{\infty} J_{3}\left(\eta_{j}, s\right), \\
\Lambda= & \Lambda_{0}+\Lambda_{1}\left(\int_{0}^{1} \tau^{\beta-r_{1}-1} h_{1}(\tau) d A_{1}(\tau)\right) \\
& +\Lambda_{2}\left(\int_{0}^{\eta} \tau^{\beta-r_{2}-1} h_{2}(\tau) d A_{2}(\tau)\right)+\Lambda_{3} \sum_{j=1}^{\infty} \rho_{j} \eta_{j}^{\beta-r_{3}-1} .
\end{aligned}
$$

Proof

(1) For $0 \leq s \leq t \leq 1$,

$$
\begin{aligned}
J_{0}(t, s)= & \frac{1}{\Gamma\left(\beta-\beta_{n-2}\right)}\left[t^{\beta-\beta_{n-2}-1}(1-s)^{\beta-r_{0}-1}-(t-s)^{\beta-\beta_{n-2}-1}\right] \\
\geq & \frac{1}{\Gamma\left(\beta-\beta_{n-2}\right)}\left[t^{\beta-\beta_{n-2}-1}(1-s)^{\beta-r_{0}-1}\right. \\
& \left.-t^{\beta-\beta_{n-2}-1}(1-s)^{\beta-\beta_{n-2}-1}\right] \\
= & \frac{1}{\Gamma\left(\beta-\beta_{n-2}\right)} t^{\beta-\beta_{n-2}-1}(1-s)^{\beta-r_{0}-1}\left[1-(1-s)^{r_{0}-\beta_{n-2}}\right] \\
= & t^{\beta-\beta_{n-2}-1} g_{0}(s), \\
J_{0}(t, s)= & \frac{1}{\Gamma\left(\beta-\beta_{n-2}\right)}\left[t^{\beta-\beta_{n-2}-1}(1-s)^{\beta-r_{0}-1}-(t-s)^{\beta-\beta_{n-2}-1}\right] \\
\leq & \Lambda_{0} t^{\beta-\beta_{n-2}-1} .
\end{aligned}
$$


For $0 \leq t \leq s \leq 1$,

$$
\begin{aligned}
J_{0}(t, s) & =\frac{1}{\Gamma\left(\beta-\beta_{n-2}\right)} t^{\beta-\beta_{n-2}-1}(1-s)^{\beta-r_{0}-1} \\
& \geq \frac{1}{\Gamma\left(\beta-\beta_{n-2}\right)} t^{\beta-\beta_{n-2}-1}(1-s)^{\beta-r_{0}-1}\left[1-(1-s)^{r_{0}-\beta_{n-2}}\right] \\
& =t^{\beta-\beta_{n-2}-1} g_{0}(s), \\
J_{0}(t, s) & =\frac{1}{\Gamma\left(\beta-\beta_{n-2}\right)} t^{\beta-\beta_{n-2}-1}(1-s)^{\beta-r_{0}-1} \\
& \leq \Lambda_{0} t^{\beta-\beta_{n-2}-1} .
\end{aligned}
$$

(2) Similarly as (1), we can obtain that

$$
t^{\beta-r_{i}-1} g_{i}(s) \leq J_{i}(t, s) \leq \Lambda_{i} t^{\beta-r_{i}-1}, \quad i=1,2,3 .
$$

(3) In view of the conclusions of (1) and (2), we have

$$
\begin{aligned}
& J(t, s)=J_{0}(t, s)+t^{\beta-\beta_{n-2}-1}\left(\int_{0}^{1} J_{1}(\tau, s) h_{1}(\tau) d A_{1}(\tau)\right) \\
& +t^{\beta-\beta_{n-2}-1}\left(\int_{0}^{\eta} J_{2}(\tau, s) h_{2}(\tau) d A_{2}(\tau)\right) \\
& +t^{\beta-\beta_{n-2}-1} \sum_{j=1}^{\infty} \rho_{j} J_{3}\left(\eta_{j}, s\right) \\
& \geq t^{\beta-\beta_{n-2}-1} g_{0}(s)+t^{\beta-\beta_{n-2}-1}\left(\int_{0}^{1} J_{1}(\tau, s) h_{1}(\tau) d A_{1}(\tau)\right) \\
& +t^{\beta-\beta_{n-2}-1}\left(\int_{0}^{\eta} J_{2}(\tau, s) h_{2}(\tau) d A_{2}(\tau)\right) \\
& +t^{\beta-\beta_{n-2}-1} \sum_{j=1}^{\infty} \rho_{j} J_{3}\left(\eta_{j}, s\right) \\
& =h(t) g(s) \text {, } \\
& J(t, s)=J_{0}(t, s)+t^{\beta-\beta_{n-2}-1}\left(\int_{0}^{1} J_{1}(\tau, s) h_{1}(\tau) d A_{1}(\tau)\right) \\
& +t^{\beta-\beta_{n-2}-1}\left(\int_{0}^{\eta} J_{2}(\tau, s) h_{2}(\tau) d A_{2}(\tau)\right) \\
& +t^{\beta-\beta_{n-2}-1} \sum_{j=1}^{\infty} \rho_{j} J_{3}\left(\eta_{j}, s\right) \\
& \leq \Lambda_{0} t^{\beta-\beta_{n-2}-1}+\Lambda_{1} t^{\beta-\beta_{n-2}-1}\left(\int_{0}^{1} \tau^{\beta-r_{1}-1} h_{1}(\tau) d A_{1}(\tau)\right) \\
& +\Lambda_{2} t^{\beta-\beta_{n-2}-1}\left(\int_{0}^{\eta} \tau^{\beta-r_{2}-1} h_{2}(\tau) d A_{2}(\tau)\right) \\
& +\Lambda_{3} t^{\beta-\beta_{n-2}-1} \sum_{j=1}^{\infty} \rho_{j} \eta_{j}^{\beta-r_{3}-1} \\
& =\Lambda h(t) \text {. }
\end{aligned}
$$

The proof is complete.
Let $(E,\|\cdot\|)$ be a Banach space, $P$ be a cone in $E$, and $\theta$ be the zero element of $E$. $P$ is said to be normal if there exists a constant $N>0$ such that

$$
\theta \leq u \leq v \Longrightarrow\|u\| \leq N\|v\|, \quad \forall u, v \in P .
$$

The smallest constant, which satisfies the above inequality, is called the normality constant of $P$. Then, $E$ is a partially ordered Banach space by $P$; that is to say,

$$
v \leq u \Longleftrightarrow u-v \in P \text {. }
$$

For $u, v \in E$, the notation $u \sim v$ shows that there exist $\lambda>0$ and $\mu>0$ such that $\lambda u \leq v \leq \mu u$. Clearly, $\sim$ is an equivalence relation in $E$. For any $h \in P \backslash\{\theta\}$, let $P_{h}=\{u \in E \mid u \sim h\}=\left\{u \in E \mid \mu h \leq u \leq \mu^{-1} h, 0<\mu<1\right\}$. Then, $P_{h} \subset P$ is a component of $P$. For more details, we suggest readers to refer $[10,11,13]$.

Definition 4 (see [11]). Let $E$ be a Banach space and $D \subset E$. The operator $T: D \times D \longrightarrow E$ is called a mixed monotone operator if $T(u, v)$ is increasing in $u \in D$ and decreasing in $v \in D$, i.e.,

$$
\begin{aligned}
& u_{1} \leq u_{2}, \\
& v_{1} \geq v_{2} \Longrightarrow T\left(u_{1}, v_{1}\right) \leq T\left(u_{2}, v_{2}\right) \text {, } \\
& \forall u_{i}, v_{i} \in D, i=1,2 .
\end{aligned}
$$

Lemma 9 (see $[13,17])$. Let $P$ be a normal cone in the Banach space $E$ and T, $S: P_{h} \times P_{h} \longrightarrow P_{h}$ be mixed monotone operators which satisfy the following:

(1) For any $l \in(0,1)$, there exists $\varphi(l) \in(l, 1]$ such that

$$
T\left(l u, l^{-1} v\right) \geq \varphi(l) T(u, v), \quad \forall u, v \in P_{h} .
$$

(2) or any $l \in(0,1), u, v \in P_{h}$,

$$
S\left(l u, l^{-1} v\right) \geq l S(u, v)
$$

(3) There exists a constant $\varrho>0$ such that for any $u, v \in P_{h}, T(u, v) \geq \varrho S(u, v)$.

Then, the equation $T(u, u)+S(u, u)=u$ has a unique fixed point $u^{*} \in P_{h}$. And for any initial values $u_{0}, v_{0} \in P_{h}$, by structuring the following sequences:

$$
\begin{array}{r}
u_{n}=T\left(u_{n-1}, v_{n-1}\right)+S\left(u_{n-1}, v_{n-1}\right), \\
v_{n}=T\left(v_{n-1}, u_{n-1}\right)+S\left(v_{n-1}, u_{n-1}\right), \\
n=1,2, \ldots,
\end{array}
$$

we have $\left\|u_{n}-u^{*}\right\| \longrightarrow 0$ and $\left\|v_{n}-u^{*}\right\| \longrightarrow 0$ in $E$, as $n \longrightarrow \infty$.

Lemma 10 (see $[13,17])$. Assume $T$ and $S$ satisfy all the conditions of Lemma 9. Ien, for any $\lambda>0$, the equation 
$\lambda T(u, u)+\lambda S(u, u)=u$ has a unique solution $u_{\lambda}^{*} \in P_{h}$, which satisfies the following:

(1) If there exists $\gamma \in(0,1)$ such that

$$
\varphi(l) \geq \frac{1}{\varrho}\left(l^{\gamma}-l\right)+l^{\gamma}, \quad \forall l \in(0,1),
$$

then $u_{\lambda}^{*}$ is continuous with respect to $\lambda \in(0,+\infty)$. That is, for any $\lambda_{0} \in(0,+\infty)$,

$$
\left\|u_{\lambda}^{*}-u_{\lambda_{0}}^{*}\right\| \longrightarrow 0, \quad \text { as } \lambda \longrightarrow \lambda_{0}
$$

(2) If

$$
\varphi(l) \geq \frac{1}{\varrho}\left(l^{1 / 2}-l\right)+l^{1 / 2}, \quad \forall l \in(0,1),
$$

then $0<\lambda_{1}<\lambda_{2}$ implies $u_{\lambda_{1}}^{*}<u_{\lambda_{2}}^{*}$.

(3) If there exists $\gamma \in(0,1 / 2)$ such that

$$
\varphi(l) \geq \frac{1}{\varrho}\left(l^{\gamma}-l\right)+l^{\gamma}, \quad \forall l \in(0,1),
$$

then

$$
\begin{aligned}
\lim _{\lambda \longrightarrow 0^{+}}\left\|u_{\lambda}^{*}\right\| & =0, \\
\lim _{\lambda \longrightarrow+\infty}\left\|u_{\lambda}^{*}\right\| & =+\infty .
\end{aligned}
$$

In this paper, we denote $E=C[0,1]$ with the norm $\|v\|=\sup |v(t)|$. Then $(E,\|\cdot\|)$ is a Banach space. Let $P=$ $\{v \in E: v(t) \geq 0, t \in[0,1]\}$ be a cone in $E$. It is easy to check that $P$ is normal in $E$ with the normality constant $N=1$.

\section{Proof of Main Results}

In this section, let $P_{h}=\{u \in E: u \sim h\}$, where $h(t)=t^{\beta-\beta_{n-2}-1}, t \in[0,1]$ (defined in Lemma 8). Then, $P_{h}$ is a component of $P$. Let us define three operators $T: P_{h} \longrightarrow P$ and $T_{1}, T_{2}: P_{h} \times P_{h} \longrightarrow P$ as follows:

$$
\begin{aligned}
(T u)(t) & =\int_{0}^{1} J(t, s) \phi_{q}\left(\int_{0}^{1} H(s, \tau) f\left(\tau, I_{0+}^{\beta_{n-2}} u(\tau), I_{0+}^{\beta_{n-2}-\beta_{1}} u(\tau), \ldots, I_{0+}^{\beta_{n-2}-\beta_{n-3}} u(\tau), u(\tau)\right) d \tau\right) d s, \\
T_{1}(u, v)(t) & =\int_{0}^{1} J(t, s) \phi_{q}\left(\int_{0}^{1} H(s, \tau) F\left(\tau, I_{0^{+}}^{\beta_{n-2}} u(\tau), I_{0^{+}}^{\beta_{n-2}-\beta_{1}} u(\tau), \ldots, I_{0^{+}}^{\beta_{n-2}-\beta_{n-3}} u(\tau), u(\tau), I_{0^{+}}^{\beta_{n-2}} v(\tau), I_{0^{+}}^{\beta_{n-2}-\beta_{1}} v(\tau), \ldots, I_{0^{+}}^{\beta_{n-2}-\beta_{n-3}} v(\tau), v(\tau)\right) d \tau\right) d s, \\
T_{2}(u, v)(t) & =\int_{0}^{1} J(t, s) \phi_{q}\left(\int_{0}^{1} H(s, \tau) G\left(\tau, I_{0^{+}}^{\beta_{n-2}} u(\tau), I_{0^{+}}^{\beta_{n-2}-\beta_{1}} u(\tau), \ldots, I_{0^{+}}^{\beta_{n-2}-\beta_{n-3}} u(\tau), u(\tau), I_{0^{+}}^{\beta_{n-2}} v(\tau), I_{0^{+}}^{\beta_{n-2}-\beta_{1}} v(\tau), \ldots, I_{0^{+}}^{\beta_{n-2}-\beta_{n-3}} v(\tau), v(\tau)\right) d \tau\right) d s .
\end{aligned}
$$

It is easy to check that $u \in C[0,1]$ is a positive solution of the problem (28)-(29) if it is a fixed point of $T$ in $P_{h}$.

Proof of Theorem 1. Obviously, by $\left(\mathbf{V}_{1}\right), \quad T u=T_{1}$ $(u, u)+T_{2}(u, u)$. And it is easy to check that $u \in C[0,1]$ is a solution of the problem (28)-(29) if it satisfies $u=T u=T_{1}(u, u)+T_{2}(u, u)$.

The first work is to prove $T_{1}, T_{2}: P_{h} \times P_{h} \longrightarrow P$ are well defined. For any $u, v \in P_{h}$, there exists a constant $\mu \in(0,1)$ such that

$$
\begin{aligned}
& \mu h(t) \leq u(t) \leq \mu^{-1} h(t), \\
& \mu h(t) \leq v(t) \leq \mu^{-1} h(t),
\end{aligned}
$$

$$
t \in[0,1] .
$$

On the other hand, it is easy to check that

$$
\begin{aligned}
I_{0^{+}}^{\beta_{n-2}} h(t) & =\frac{1}{\Gamma\left(\beta_{n-2}\right)} \int_{0}^{t}(t-s)^{\beta_{n-2}-1} s^{\beta-\beta_{n-2}-1} d s \\
& =\frac{\Gamma\left(\beta-\beta_{n-2}\right)}{\Gamma(\beta)} t^{\beta-1} \leq 1, \quad t \in[0,1],
\end{aligned}
$$

$$
\begin{aligned}
I_{0^{+}}^{\beta_{n-2}-\beta_{i}} h(t)= & \frac{1}{\Gamma\left(\beta_{n-2}-\beta_{i}\right)} \int_{0}^{t}(t-s)^{\beta_{n-2}-\beta_{i}-1} s^{\beta-\beta_{n-2}-1} d s \\
= & \frac{\Gamma\left(\beta-\beta_{n-2}\right)}{\Gamma\left(\beta-\beta_{i}\right)} t^{\beta-\beta_{i}-1} \leq 1, \\
& \quad i=1,2, \ldots, n-3, t \in[0,1] .
\end{aligned}
$$

Let

$$
r=\min \left\{\frac{\Gamma\left(\beta-\beta_{n-2}\right)}{\Gamma(\beta)}, \frac{\Gamma\left(\beta-\beta_{n-2}\right)}{\Gamma\left(\beta-\beta_{1}\right)}, \ldots, \frac{\Gamma\left(\beta-\beta_{n-2}\right)}{\Gamma\left(\beta-\beta_{n-3}\right)}, 1\right\} .
$$


Then, $0<r \leq 1$. According to $\left(\mathbf{V}_{1}\right)-\left(\mathbf{V}_{3}\right)$ and (87)-(89), we have

$$
\begin{aligned}
& F\left(\tau, I_{0^{+}}^{\beta_{n-2}} u(\tau), I_{0^{+}}^{\beta_{n-2}-\beta_{1}} u(\tau), \ldots, I_{0^{+}}^{\beta_{n-2}-\beta_{n-3}} u(\tau), u(\tau), I_{0^{+}}^{\beta_{n-2}} v(\tau), I_{0^{+}}^{\beta_{n-2}-\beta_{1}} v(\tau), \ldots, I_{0^{+}}^{\beta_{n-2}-\beta_{n-3}} v(\tau), v(\tau)\right) \\
& \leq F\left(\tau, I_{0^{+}}^{\beta_{n-2}} \mu^{-1} h(\tau), I_{0^{+}}^{\beta_{n-2}-\beta_{1}} \mu^{-1} h(\tau), \ldots, I_{0^{+}}^{\beta_{n-2}-\beta_{n-3}} \mu^{-1} h(\tau), \mu^{-1} h(\tau), I_{0^{+}}^{\beta_{n-2}} \mu h(\tau), I_{0^{+}}^{\beta_{n-2}-\beta_{1}} \mu h(\tau), \ldots, I_{0^{+}}^{\beta_{n-2}-\beta_{n-3}} \mu h(\tau), \mu h(\tau)\right) \\
& \leq F\left(\tau, \mu^{-1}, \mu^{-1}, \ldots, \mu^{-1}, \mu^{-1}, \mu \frac{\Gamma\left(\beta-\beta_{n-2}\right)}{\Gamma(\beta)} \tau^{\beta-1}, \mu \frac{\Gamma\left(\beta-\beta_{n-2}\right)}{\Gamma\left(\beta-\beta_{1}\right)} \tau^{\beta-\beta_{1}-1}, \ldots, \mu \frac{\Gamma\left(\beta-\beta_{n-2}\right)}{\Gamma\left(\beta-\beta_{n-3}\right)} \tau^{\beta-\beta_{n-3}-1}, \tau^{\beta-\beta_{n-2}-1}\right) \\
& \leq F\left(\tau,(\mu r)^{-1},(\mu r)^{-1}, \ldots,(\mu r)^{-1},(\mu r)^{-1}, \mu r \tau^{\beta-1}, \mu r \tau^{\beta-\beta_{1}-1}, \ldots, \mu r \tau^{\beta-\beta_{n-3}-1}, \mu r \tau^{\beta-\beta_{n-2}-1}\right) \\
& \leq \frac{1}{\varphi^{p-1}(\mu r)} F\left(\tau, 1,1, \ldots, 1,1, \tau^{\beta-1}, \tau^{\beta-\beta_{1}-1}, \ldots, \tau^{\beta-\beta_{n-3}-1}, \tau^{\beta-\beta_{n-2}-1}\right) \\
& \leq \frac{1}{\varphi^{p^{-1}}(\mu r)} F\left(\tau, 1, \ldots, 1, \tau^{\beta-1}, \ldots, \tau^{\beta-1}\right) \\
& F\left(\tau, I_{0^{+}}^{\beta_{n-2}} u(\tau), I_{0^{+}}^{\beta_{n-2}-\beta_{1}} u(\tau), \ldots, I_{0^{+}}^{\beta_{n-2}-\beta_{n-3}} u(\tau), u(\tau), I_{0^{+}}^{\beta_{n-2}} v(\tau), I_{0^{+}}^{\beta_{n-2}-\beta_{1}} v(\tau), \ldots, I_{0^{+}}^{\beta_{n-2}-\beta_{n-3}} v(\tau), v(\tau)\right) \\
& \geq F\left(\tau, I_{0^{+}}^{\beta_{n-2}} \mu h(\tau), I_{0^{+}}^{\beta_{n-2}-\beta_{1}} \mu h(\tau), \ldots, I_{0^{+}}^{\beta_{n-2}-\beta_{n-3}} \mu h(\tau), \mu h(\tau), I_{0^{+}}^{\beta_{n-2}} \mu^{-1} h(\tau), I_{0^{+}}^{\beta_{n-2}-\beta_{1}} \mu^{-1} h(\tau), \ldots, I_{0^{+}}^{\beta_{n-2}-\beta_{n-3}} \mu^{-1} h(\tau), \mu^{-1} h(\tau)\right) \\
& \geq F\left(\tau, \mu \frac{\Gamma\left(\beta-\beta_{n-2}\right)}{\Gamma(\beta)} \tau^{\beta-1}, \mu \frac{\Gamma\left(\beta-\beta_{n-2}\right)}{\Gamma\left(\beta-\beta_{1}\right)} \tau^{\beta-\beta_{1}-1}, \ldots, \mu \frac{\Gamma\left(\beta-\beta_{n-2}\right)}{\Gamma\left(\beta-\beta_{n-3}\right)} \tau^{\beta-\beta_{n-3}-1}, \mu \tau^{\beta-\beta_{n-2}-1}, \mu^{-1}, \mu^{-1}, \ldots, \mu^{-1}, \mu^{-1}\right) \\
& \geq F\left(\tau, \mu r \tau^{\beta-1}, \mu r \tau^{\beta-\beta_{1}-1}, \ldots, \mu r \tau^{\beta-\beta_{n-3}-1}, \mu r \tau^{\beta-\beta_{n-2}-1},(\mu r)^{-1},(\mu r)^{-1}, \ldots,(\mu r)^{-1},(\mu r)^{-1}\right) \\
& \geq \varphi^{p-1}(\mu r) F\left(\tau, \tau^{\beta-1}, \tau^{\beta-\beta_{1}-1}, \ldots, \tau^{\beta-\beta_{n-3}-1}, \tau^{\beta-\beta_{n-2}-1}, 1,1, \ldots, 1,1\right) \\
& \geq \varphi^{p-1}(\mu r) F\left(\tau, \tau^{\beta-1}, \ldots, \tau^{\beta-1}, 1, \ldots, 1\right) \text {. }
\end{aligned}
$$

Similarly, we have

$$
\begin{aligned}
& G\left(\tau, I_{0^{+}}^{\beta_{n-2}} u(\tau), I_{0^{+}}^{\beta_{n-2}-\beta_{1}} u(\tau), \ldots, I_{0^{+}}^{\beta_{n-2}-\beta_{n-3}} u(\tau),\right. \\
& \left.u(\tau), I_{0^{+}}^{\beta_{n-2}} v(\tau), I_{0^{+}}^{\beta_{n-2}-\beta_{1}} v(\tau), \ldots, I_{0^{+}}^{\beta_{n-2}-\beta_{n-3}} v(\tau), v(\tau)\right) \\
& \quad \leq \frac{1}{(\mu r)^{p-1}} G\left(\tau, 1, \ldots, 1, \tau^{\beta-1}, \ldots, \tau^{\beta-1}\right),
\end{aligned}
$$

$G\left(\tau, I_{0^{+}}^{\beta_{n-2}} u(\tau), I_{0^{+}}^{\beta_{n-2}-\beta_{1}} u(\tau), \ldots, I_{0^{+}}^{\beta_{n-2}-\beta_{n-3}} u(\tau), u(\tau), I_{0^{+}}^{\beta_{n-2}} v(\tau)\right.$, $\left.I_{0^{+}}^{\beta_{n-2}-\beta_{1}} v(\tau), \ldots, I_{0^{+}}^{\beta_{n-2}-\beta_{n-3}} v(\tau), v(\tau)\right) \geq(\mu r)^{p^{-1}}$ $\cdot G\left(\tau, \tau^{\beta-1}, \ldots, \tau^{\beta-1}, 1, \ldots, 1\right)$.

On the basis of (90), (92), and $\left(\mathbf{V}_{5}\right)$, we know that for any $t \in[0,1]$,

$$
\begin{aligned}
T_{1}(u, v)(t) & =\int_{0}^{1} J(t, s) \phi_{q}\left(\int_{0}^{1} H(s, \tau) F\left(\tau, I_{0^{+}}^{\beta_{n-2}} u(\tau), I_{0^{+}}^{\beta_{n-2}-\beta_{1}} u(\tau), \ldots, I_{0^{+}}^{\beta_{n-2}-\beta_{n-3}} u(\tau), u(\tau), I_{0^{+}}^{\beta_{n-2}} v(\tau), I_{0^{+}}^{\beta_{n-2}-\beta_{1}} v(\tau), \ldots, I_{0^{+}}^{\beta_{n-2}-\beta_{n-3}} v(\tau), v(\tau)\right) d \tau\right) d s \\
& \leq \frac{\Lambda h(t)}{(\Gamma(\alpha))^{q-1} \varphi(\mu r)} \int_{0}^{1} \phi_{q}\left(\int_{0}^{1}(1-\tau)^{\alpha-1} F\left(\tau, 1, \ldots, 1, \tau^{\beta-1}, \ldots, \tau^{\beta-1}\right) d \tau\right) d s \\
& <+\infty
\end{aligned}
$$

$T_{2}(u, v)(t) \leq \frac{\Lambda h(t)}{(\Gamma(\alpha))^{q-1}(\mu r)} \int_{0}^{1} \phi_{q}\left(\int_{0}^{1}(1-\tau)^{\alpha-1} G\left(\tau, 1, \ldots, 1, \tau^{\beta-1}, \ldots, \tau^{\beta-1}\right) d \tau\right) d s<+\infty$ 
That is, $T_{1}, T_{2}: P_{h} \times P_{h} \longrightarrow P$ are well defined. On the other hand, by means of $\left(\mathbf{V}_{2}\right)$, for any $\left(u_{1}, v_{1}\right),\left(u_{2}, v_{2}\right)$ satisfying $u_{1} \leq u_{2}$ and $v_{1} \geq v_{2}$, we have

$$
\begin{aligned}
T_{1}\left(u_{1}, v_{1}\right)(t)= & \int_{0}^{1} J(t, s) \phi_{q}\left(\int _ { 0 } ^ { 1 } H ( s , \tau ) F \left(\tau, I_{0^{+}}^{\beta_{n-2}} u_{1}(\tau), I_{0^{+}}^{\beta_{n-2}-\beta_{1}} u_{1}(\tau), \ldots, I_{0^{+}}^{\beta_{n-2}-\beta_{n-3}} u_{1}(\tau), u_{1}(\tau), I_{0^{+}}^{\beta_{n-2}} v_{1}(\tau),\right.\right. \\
& \left.\left.I_{0^{+}}^{\beta_{n-2}-\beta_{1}} v_{1}(\tau), \ldots, I_{0^{+}}^{\beta_{n-2}-\beta_{n-3}} v_{1}(\tau), v_{1}(\tau)\right) d \tau\right) d s \\
\leq & \int_{0}^{1} J(t, s) \phi_{q}\left(\int _ { 0 } ^ { 1 } H ( s , \tau ) F \left(\tau, I_{0^{+}}^{\beta_{n-2}} u_{2}(\tau), I_{0^{+}}^{\beta_{n-2}-\beta_{1}} u_{2}(\tau), \ldots, I_{0^{+}}^{\beta_{n-2}-\beta_{n-3}} u_{2}(\tau), u_{2}(\tau), I_{0^{+}}^{\beta_{n-2}} v_{2}(\tau),\right.\right. \\
& \left.\left.I_{0^{+}}^{\beta_{n-2}-\beta_{1}} v_{2}(\tau), \ldots, I_{0^{+}}^{\beta_{n-2}-\beta_{n-3}} v_{2}(\tau), v_{2}(\tau)\right) d \tau\right) d s \\
= & T_{1}\left(u_{2}, v_{2}\right)(t), t \in[0,1] \\
T_{2}\left(u_{1}, v_{1}\right)(t)= & \int_{0}^{1} J(t, s) \phi_{q}\left(\int_{0}^{1} H(s, \tau) G \tau, I_{0^{+}}^{\beta_{n-2}} u_{1}(\tau), I_{0^{+}}^{\beta_{n-2}-\beta_{1}} u_{1}(\tau), \ldots, I_{0^{+}}^{\beta_{n-2}-\beta_{n-3}} u_{1}(\tau), u_{1}(\tau), I_{0^{+}}^{\beta_{n-2}} v_{1}(\tau),\right. \\
& \left.I_{0^{+}}^{\beta_{n-2}-\beta_{1}} v_{1}(\tau), \ldots, I_{0^{+}}^{\beta_{n-2}-\beta_{n-3}} v_{1}(\tau), v_{1}(\tau) d \tau\right) d s \\
\leq & \int_{0}^{1} J(t, s) \phi_{q}\left(\int _ { 0 } ^ { 1 } H ( s , \tau ) G \left(\tau, I_{0^{+}}^{\beta_{n-2}} u_{2}(\tau), I_{0^{+}}^{\beta_{n-2}-\beta_{1}} u_{2}(\tau), \ldots, I_{0^{+}}^{\beta_{n-2}-\beta_{n-3}} u_{2}(\tau), u_{2}(\tau), I_{0^{+}}^{\beta_{n-2}} v_{2}(\tau),\right.\right. \\
& \left.\left.I_{0^{+}}^{\beta_{n-2}-\beta_{1}} v_{2}(\tau), \ldots, I_{0^{+}}^{\beta_{n-2}-\beta_{n-3}} v_{2}(\tau), v_{2}(\tau)\right) d \tau\right) d s \\
= & T_{2}\left(u_{2}, v_{2}\right)(t), t \in[0,1] .
\end{aligned}
$$

Hence, $T_{1}, T_{2}: P_{h} \times P_{h} \longrightarrow P$ are mixed monotone operators.
In the following, we prove that $T_{1}, T_{2}: P_{h} \times P_{h} \longrightarrow P_{h}$. It follows from (91) and (93) that for any $u, v \in P_{h}$ and $t \in[0,1]$

$$
\begin{aligned}
T_{1}(u, v)(t)= & \int_{0}^{1} J(t, s) \phi_{q}\left(\int _ { 0 } ^ { 1 } H ( s , \tau ) F \left(\tau, I_{0^{+}}^{\beta_{n-2}} u(\tau), I_{0^{+}}^{\beta_{n-2}-\beta_{1}} u(\tau), \ldots, I_{0^{+}}^{\beta_{n-2}-\beta_{n-3}} u(\tau), u(\tau), I_{0^{+}}^{\beta_{n-2}} v(\tau),\right.\right. \\
& \left.\left.I_{0^{+}}^{\beta_{n-2}-\beta_{1}} v(\tau), \ldots, I_{0^{+}}^{\beta_{n-2}-\beta_{n-3}} v(\tau), v(\tau)\right) d \tau\right) d s \\
\geq & \int_{0}^{1} J(t, s) \phi_{q}\left(\varphi^{p-1}(\mu r) \int_{0}^{1} H(s, \tau) F\left(\tau, \tau^{\beta-1}, \ldots, \tau^{\beta-1}, 1, \ldots, 1\right) d \tau\right) d s \\
\geq & \varphi(\mu r) h(t) \int_{0}^{1} g(s) \phi_{q}\left(\int_{0}^{1} H(s, \tau) F\left(\tau, \tau^{\beta-1}, \ldots, \tau^{\beta-1}, 1, \ldots, 1\right) d \tau\right) d s \\
\geq & M^{-1} h(t), \\
T_{2}(u, v)(t) \geq & \mu r h(t) \int_{0}^{1} g(s) \phi_{q}\left(\int_{0}^{1} H(s, \tau) G\left(\tau, \tau^{\beta-1}, \ldots, \tau^{\beta-1}, 1, \ldots, 1\right) d \tau\right) d s \\
\geq & M^{-1} h(t),
\end{aligned}
$$

where $M$ is a constant, which satisfies 


$$
\begin{gathered}
\max \left\{\frac{\Lambda}{(\Gamma(\alpha))^{q-1} \varphi(\mu r)} \int_{0}^{1} \phi_{q}\left(\int_{0}^{1}(1-\tau)^{\alpha-1} F\left(\tau, 1, \ldots, 1, \tau^{\beta-1}, \ldots, \tau^{\beta-1}\right) d \tau\right) d s,\right. \\
\cdot \frac{\Lambda}{(\Gamma(\alpha))^{q-1}(\mu r)} \int_{0}^{1} \phi_{q}\left(\int_{0}^{1}(1-\tau)^{\alpha-1} G\left(\tau, 1, \ldots, 1, \tau^{\beta-1}, \ldots, \tau^{\beta-1}\right) d \tau\right) d s \\
\cdot\left(\varphi(\mu r) \int_{0}^{1} g(s) \phi_{q}\left(\int_{0}^{1} H(s, \tau) F\left(\tau, \tau^{\beta-1}, \ldots, \tau^{\beta-1}, 1, \ldots, 1\right) d \tau\right) d s\right)^{-1}, \\
\left.\cdot\left(\mu r \int_{0}^{1} g(s) \phi_{q}\left(\int_{0}^{1} H(s, \tau) G\left(\tau, \tau^{\beta-1}, \ldots, \tau^{\beta-1}, 1, \ldots, 1\right) d \tau\right) d s\right)^{-1}\right\}<M
\end{gathered}
$$

According to (94) and (95), we have

$$
\begin{aligned}
T_{1}(u, v)(t) & \leq \frac{\Lambda h(t)}{(\Gamma(\alpha))^{q-1} \varphi(\mu r)} \int_{0}^{1} \phi_{q}\left(\int_{0}^{1}(1-\tau)^{\alpha-1} F\left(\tau, 1, \ldots, 1, \tau^{\beta-1}, \ldots, \tau^{\beta-1}\right) d \tau\right) d s \\
& \leq M h(t), \\
T_{2}(u, v)(t) & \leq \frac{\Lambda h(t)}{(\Gamma(\alpha))^{q-1}(\mu r)} \int_{0}^{1} \phi_{q}\left(\int_{0}^{1}(1-\tau)^{\alpha-1} G\left(\tau, 1, \ldots, 1, \tau^{\beta-1}, \ldots, \tau^{\beta-1}\right) d \tau\right) d s \\
& \leq M h(t) .
\end{aligned}
$$

On the basis of (98), (99), (101), and (102) we infer that $T_{1}, T_{2}: P_{h} \times P_{h} \longrightarrow P_{h}$.
Moreover, it follows from $\left(\mathbf{V}_{3}\right)$, for any $l \in(0,1)$ and $u$, $v \in P_{h}$ and $t \in[0,1]$, we have

$$
\begin{aligned}
T_{1}\left(l u, l^{-1} v\right)(t)= & \int_{0}^{1} J(t, s) \phi_{q}\left(\int _ { 0 } ^ { 1 } H ( s , \tau ) F \left(\tau, I_{0^{+}}^{\beta_{n-2}} l u(\tau), I_{0^{+}}^{\beta_{n-2}-\beta_{1}} l u(\tau), \ldots, I_{0^{+}}^{\beta_{n-2}-\beta_{n-3}} l u(\tau), l u(\tau), I_{0^{+}}^{\beta_{n-2}} l^{-1} v(\tau),\right.\right. \\
& \left.\left.I_{0^{+}}^{\beta_{n-2}-\beta_{1}} l^{-1} v(\tau), \ldots, I_{0^{+}}^{\beta_{n-2}-\beta_{n-3}} l^{-1} v(\tau), l^{-1} v(\tau)\right) d \tau\right) d s \\
= & \int_{0}^{1} J(t, s) \phi_{q}\left(\int _ { 0 } ^ { 1 } H ( s , \tau ) F \left(\tau, l I_{0^{+}}^{\beta_{n-2}} u(\tau), l I_{0^{+}}^{\beta_{n-2}-\beta_{1}} u(\tau), \ldots, l I_{0^{+}}^{\beta_{n-2}-\beta_{n-3}} u(\tau), l u(\tau), l^{-1} I_{0^{+}}^{\beta_{n-2}} v(\tau),\right.\right. \\
& \left.\left.l^{-1} I_{0^{+}}^{\beta_{n-2}-\beta_{1}} v(\tau), \ldots, l^{-1} I_{0^{+}}^{\beta_{n-2}-\beta_{n-3}} v(\tau), l^{-1} v(\tau)\right) d \tau\right) d s \\
\geq & \int_{0}^{1} J(t, s) \phi_{q} \varphi^{p-1}(l)\left(\int _ { 0 } ^ { 1 } H ( s , \tau ) F \left(\tau, I_{0^{+}}^{\beta_{n-2}} u(\tau), I_{0^{+}}^{\beta_{n-2}-\beta_{1}} u(\tau), \ldots, I_{0^{+}}^{\beta_{n-2}-\beta_{n-3}} u(\tau), u(\tau), I_{0^{+}}^{\beta_{n-2}} v(\tau),\right.\right. \\
& \left.\left.I_{0^{+}}^{\beta_{n-2}-\beta_{1}} v(\tau), \ldots, I_{0^{+}}^{\beta_{n-2}-\beta_{n-3}} v(\tau), v(\tau)\right) d \tau\right) d s \\
= & \varphi(l) \int_{0}^{1} J(t, s) \phi_{q}\left(\int _ { 0 } ^ { 1 } H ( s , \tau ) F \left(\tau, I_{0^{+}}^{\beta_{n-2}} u(\tau), I_{0^{+}}^{\beta_{n-2}-\beta_{1}} u(\tau), \ldots, I_{0^{+}}^{\beta_{n-2}-\beta_{n-3}} u(\tau), u(\tau), I_{0^{+}}^{\beta_{n-2}} v(\tau),\right.\right. \\
& \left.\left.I_{0^{+}}^{\beta_{n-2}-\beta_{1}} v(\tau), \ldots, I_{0^{+}}^{\beta_{n-2}-\beta_{n-3}} v(\tau), v(\tau)\right) d \tau\right) d s \\
= & \varphi(l) T_{1}(u, v)(t),
\end{aligned}
$$




$$
\begin{aligned}
T_{2}\left(l u, l^{-1} v\right)(t)= & \int_{0}^{1} J(t, s) \phi_{q}\left(\int _ { 0 } ^ { 1 } H ( s , \tau ) G \left(\tau, I_{0^{+}}^{\beta_{n-2}} l u(\tau), I_{0^{+}}^{\beta_{n-2}-\beta_{1}} l u(\tau), \ldots, I_{0^{+}}^{\beta_{n-2}-\beta_{n-3}} l u(\tau), l u(\tau), I_{0^{+}}^{\beta_{n-2}} l^{-1} v(\tau)\right.\right. \\
& \left.\left.I_{0^{+}}^{\beta_{n-2}-\beta_{1}} l^{-1} v(\tau), \ldots, I_{0^{+}}^{\beta_{n-2}-\beta_{n-3}} l^{-1} v(\tau), l^{-1} v(\tau)\right) d \tau\right) d s \\
\geq & l \int_{0}^{1} J(t, s) \phi_{q}\left(\int _ { 0 } ^ { 1 } H ( s , \tau ) G \left(\tau, I_{0^{+}}^{\beta_{n-2}} u(\tau), I_{0^{+}}^{\beta_{n-2}-\beta_{1}} u(\tau), \ldots, I_{0^{+}}^{\beta_{n-2}-\beta_{n-3}} u(\tau), u(\tau), I_{0^{+}}^{\beta_{n-2}} v(\tau)\right.\right. \\
& \left.\left.I_{0^{+}}^{\beta_{n-2}-\beta_{1}} v(\tau), \ldots, I_{0^{+}}^{\beta_{n-2}-\beta_{n-3}} v(\tau), v(\tau)\right) d \tau\right) d s \\
= & l T_{2}(u, v)(t) .
\end{aligned}
$$

From $\left(\mathbf{V}_{4}\right)$, for any $u, v \in P_{h}$ and $t \in[0,1]$, we can obtain

$$
\begin{aligned}
T_{1}(u, v)(t)= & \int_{0}^{1} J(t, s) \phi_{q}\left(\int _ { 0 } ^ { 1 } H ( s , \tau ) F \left(\tau, I_{0^{+}}^{\beta_{n-2}} u(\tau), I_{0^{+}}^{\beta_{n-2}-\beta_{1}} u(\tau), \ldots, I_{0^{+}}^{\beta_{n-2}-\beta_{n-3}} u(\tau), u(\tau), I_{0^{+}}^{\beta_{n-2}} v(\tau),\right.\right. \\
& \left.\left.I_{0^{+}}^{\beta_{n-2}-\beta_{1}} v(\tau), \ldots, I_{0^{+}}^{\beta_{n-2}-\beta_{n-3}} v(\tau), v(\tau)\right) d \tau\right) d s \\
\geq & \int_{0}^{1} J(t, s) \phi_{q}\left(\varrho ^ { p - 1 } \int _ { 0 } ^ { 1 } H ( s , \tau ) G \left(\tau, I_{0^{+}}^{\beta_{n-2}} u(\tau), I_{0^{+}}^{\beta_{n-2}-\beta_{1}} u(\tau), \ldots, I_{0^{+}}^{\beta_{n-2}-\beta_{n-3}} u(\tau), u(\tau), I_{0^{+}}^{\beta_{n-2}} v(\tau),\right.\right. \\
& \left.\left.\left.I_{0^{+}}^{\beta_{n-2}-\beta_{1}} v(\tau), \ldots, I_{0^{+}}^{\beta_{n-2}-\beta_{n-3}} v(\tau), v(\tau)\right)\right) d \tau\right) d s \\
= & \varrho T_{2}(u, v)(t) .
\end{aligned}
$$

So, based on (103)-(105) and Lemma 9, we know the $\delta t^{\beta-\beta_{n-2}-1}=\delta h(t) \leq u^{*}(t) \leq \delta^{-1} h(t)=\delta^{-1} t^{\beta-\beta_{n-2}-1}, \quad t \in[0,1]$. equation $T_{1}(u, u)+T_{2}(u, u)=u$ has a unique fixed point $u^{*} \in P_{h}$, which means $u^{*}$ is the unique positive solution of the problem (28)-(29), and there exists $\delta \in(0,1)$ such that

And for any initial values $u_{0}, \tilde{u}_{0} \in P_{h}$, we can construct the following sequences:

$$
\begin{aligned}
u_{n}(t)= & \int_{0}^{1} J(t, s) \phi_{q}\left(\int _ { 0 } ^ { 1 } H ( s , \tau ) F \left(\tau, I_{0^{+}}^{\beta_{n-2}} u_{n-1}(\tau), I_{0^{+}}^{\beta_{n-2}-\beta_{1}} u_{n-1}(\tau), \ldots, I_{0^{+}}^{\beta_{n-2}-\beta_{n-3}} u_{n-1}(\tau), u_{n-1}(\tau), I_{0^{+}}^{\beta_{n-2}} \widetilde{u}_{n-1}(\tau),\right.\right. \\
& \left.\left.I_{0^{+}}^{\beta_{n-2}-\beta_{1}} \widetilde{u}_{n-1}(\tau), \ldots, I_{0^{+}}^{\beta_{n-2}-\beta_{n-3}} \widetilde{u}_{n-1}(\tau), \widetilde{u}_{n-1}(\tau)\right) d \tau\right) d s \\
+ & \int_{0}^{1} J(t, s) \phi_{q}\left(\int _ { 0 } ^ { 1 } H ( s , \tau ) G \left(\tau, I_{0^{+}}^{\beta_{n-2}} u_{n-1}(\tau), I_{0^{+}}^{\beta_{n-2}-\beta_{1}} u_{n-1}(\tau), \ldots, I_{0^{+}}^{\beta_{n-2}-\beta_{n-3}} u_{n-1}(\tau), u_{n-1}(\tau), I_{0^{+}}^{\beta_{n-2}} \widetilde{u}_{n-1}(\tau)\right.\right. \\
& \left.\left.I_{0^{+}}^{\beta_{n-2}-\beta_{1}} \widetilde{u}_{n-1}(\tau), \ldots, I_{0^{+}}^{\beta_{n-2}-\beta_{n-3}} \widetilde{u}_{n-1}(\tau), \widetilde{u}_{n-1}(\tau)\right) d \tau\right) d s \\
\widetilde{u}_{n}(t)= & \int_{0}^{1} J(t, s) \phi_{q}\left(\int _ { 0 } ^ { 1 } H ( s , \tau ) F \left(\tau, I_{0^{+}}^{\beta_{n-2}} \widetilde{u}_{n-1}(\tau), I_{0^{+}}^{\beta_{n-2}-\beta_{1}} \widetilde{u}_{n-1}(\tau), \ldots, I_{0^{+}}^{\beta_{n-2}-\beta_{n-3}} \widetilde{u}_{n-1}(\tau), \widetilde{u}_{n-1}(\tau)\right.\right. \\
& \left.\left.I_{0^{+}}^{\beta_{n-2}} u_{n-1}(\tau), I_{0^{+}-2}^{\beta_{n-}} u_{n-1}(\tau), \ldots, I_{0^{+}}^{\beta_{n-2}-\beta_{n-3}} u_{n-1}(\tau), u_{n-1}(\tau)\right) d \tau\right) d s \\
+ & \int_{0}^{1} J(t, s) \phi_{q}\left(\int _ { 0 } ^ { 1 } H ( s , \tau ) G \left(\tau, I_{0^{+}}^{\beta_{n-2}} \widetilde{u}_{n-1}(\tau), I_{0^{+}}^{\beta_{n-2}-\beta_{1}} \widetilde{u}_{n-1}(\tau), \ldots, I_{0^{+}}^{\beta_{n-2}-\beta_{n-3}} \widetilde{u}_{n-1}(\tau), \widetilde{u}_{n-1}(\tau)\right.\right. \\
& \left.\left.I_{0^{+}}^{\beta_{n-2}} u_{n-1}(\tau), I_{0^{+}}^{\beta_{n-2}-\beta_{1}} u_{n-1}(\tau), \ldots, I_{0^{+}}^{\beta_{n-2}-\beta_{n-3}} u_{n-1}(\tau), u_{n-1}(\tau)\right) d \tau\right) d s
\end{aligned}
$$

$t \in[0,1], \quad n=1,2, \ldots$,

such that 


$$
\begin{array}{r}
\sup _{t \in[0,1]}\left|u_{n}(t)-u^{*}(t)\right|=\left\|u_{n}-u^{*}\right\| \longrightarrow 0 \\
\sup _{t \in[0,1]}\left|\tilde{u}_{n}(t)-u^{*}(t)\right|=\left\|\widetilde{u}_{n}-u^{*}\right\| \longrightarrow 0 \\
\text { as } n \longrightarrow \infty .
\end{array}
$$

Finally, on the basis of Lemma 5, we know $v^{*}=I^{\beta_{n-2}} u^{*}$ is the unique positive solution of problem (4)-(5). By (106), we have

$$
\frac{\Gamma\left(\beta-\beta_{n-2}\right) \delta}{\Gamma(\beta)} t^{\beta-1} \leq v^{*}(t) \leq \frac{\Gamma\left(\beta-\beta_{n-2}\right)}{\Gamma(\beta) \delta} t^{\beta-1}, \quad t \in[0,1] .
$$

Let $v_{n}=I_{0^{+}}^{\beta_{n-2}} u_{n}$ and $\widetilde{v}_{n}=I_{0^{+}}^{\beta_{n-2}} \widetilde{u}_{n}$; by using the monotonicity and continuity of fractional integral, we have

$$
\begin{aligned}
\sup _{t \in[0,1]}\left|v_{n}(t)-v^{*}(t)\right|=\left\|v_{n}-v^{*}\right\| & \longrightarrow 0, \\
\sup _{t \in[0,1]}\left|\widetilde{v}_{n}(t)-v^{*}(t)\right|=\left\|\widetilde{v}_{n}-v^{*}\right\| & \longrightarrow 0, \\
\text { as } n & \longrightarrow \infty .
\end{aligned}
$$

Proof of Theorem 2. For any $\lambda>0$, let us define three operators $\widetilde{T}, \widetilde{T}_{1}, \widetilde{T}_{2}: P_{h} \times P_{h} \longrightarrow P$ by $\widetilde{T}=\left(\lambda^{q-1} T\right), \widetilde{T}_{1}=\left(\lambda^{q-1} T_{1}\right)$, and $\widetilde{T}_{2}=\left(\lambda^{q-1} T_{2}\right)$, respectively. It is easy to check that $u \in C[0,1]$ is a positive solution of the problem (44)-(29) if it is a fixed point of $\widetilde{T}$ in $P_{h}$, i.e., $u=\widetilde{T} u=\widetilde{T}_{1}(u, u)+\widetilde{T}_{2}(u, u)$. Considering the results of Theorem 1 and Lemma 10 together, we know the following equation

$$
\widetilde{T}_{1}(u, u)+\widetilde{T}_{2}(u, u)=\left(\lambda^{q-1} T_{1}\right)(u, u)+\left(\lambda^{q-1} T_{2}\right)(u, u)=u,
$$

has a unique solution $u_{\lambda}^{*} \in P_{h}$, which implies $u_{\lambda}^{*}$ is the unique positive solution of the problem (44)-(29), and there exists $\delta_{\lambda} \in(0,1)$ such that

$$
\delta_{\lambda} t^{\beta-\beta_{n-2}-1}=\delta_{\lambda} h(t) \leq u_{\lambda}^{*}(t) \leq \delta_{\lambda}^{-1} h(t)=\delta_{\lambda}^{-1} t^{\beta-\beta_{n-2}-1}, \quad t \in[0,1] .
$$

Furthermore, $u_{\lambda}^{*}$ satisfies the following conclusion:

(1) If there exists $\gamma \in(0,1)$ such that

$$
\varphi(l) \geq \frac{1}{\varrho}\left(l^{\gamma}-l\right)+l^{\gamma}, \quad \forall l \in(0,1)
$$

then $u_{\lambda}^{*}$ is continuous with respect to $\lambda \in(0,+\infty)$. That is, for any $\lambda_{0} \in(0,+\infty)$,

$$
\sup _{t \in[0,1]}\left|u_{\lambda}^{*}(t)-u_{\lambda_{0}}^{*}(t)\right|=\left\|u_{\lambda}^{*}-u_{\lambda_{0}}^{*}\right\| \longrightarrow 0, \quad \text { as } \lambda \longrightarrow \lambda_{0} \text {. }
$$

(2) If

$$
\varphi(l) \geq \frac{1}{\varrho}\left(l^{1 / 2}-l\right)+l^{1 / 2}, \quad \forall l \in(0,1),
$$

then $0<\lambda_{1}<\lambda_{2}$ implies $u_{\lambda_{1}}^{*}<u_{\lambda_{2}}^{*}$.

(3) If there exists $\gamma \in(0,1 / 2)$ such that

$$
\varphi(l) \geq \frac{1}{\varrho}\left(l^{\gamma}-l\right)+l^{\gamma}, \quad \forall l \in(0,1),
$$

then

$$
\begin{aligned}
& \lim _{\lambda \longrightarrow 0^{+}} \sup _{t \in[0,1]}\left|u_{\lambda}^{*}(t)\right|=\lim _{\lambda \longrightarrow 0^{+}}\left\|u_{\lambda}^{*}\right\|=0, \\
& \lim _{\lambda \longrightarrow+\infty} \sup _{t \in[0,1]}\left|u_{\lambda}^{*}(t)\right|=\lim _{\lambda \longrightarrow+\infty}\left\|u_{\lambda}^{*}\right\|=+\infty .
\end{aligned}
$$

Finally, by Lemma 5 , we deduce $v_{\lambda}^{*}=I_{0^{+}}^{\beta_{n-2}} u_{\lambda}^{*}$ is a unique positive solution of the problem (6)-(5), which satisfies

$$
\frac{\Gamma\left(\beta-\beta_{n-2}\right) \delta_{\lambda}}{\Gamma(\beta)} t^{\beta-1} \leq v_{\lambda}^{*}(t) \leq \frac{\Gamma\left(\beta-\beta_{n-2}\right)}{\Gamma(\beta) \delta_{\lambda}} t^{\beta-1}, \quad t \in[0,1] .
$$

And by using the monotonicity and continuity of fractional integral, we have the following:

(1) If there exists $\gamma \in(0,1)$ such that

$$
\varphi(l) \geq \frac{1}{\varrho}\left(l^{\gamma}-l\right)+l^{\gamma}, \quad \forall l \in(0,1),
$$

then $v_{\lambda}^{*}$ is continuous with respect to $\lambda \in(0,+\infty)$. That is, for any $\lambda_{0} \in(0,+\infty)$,

$$
\sup _{t \in[0,1]}\left|v_{\lambda}^{*}(t)-v_{\lambda_{0}}^{*}(t)\right|=\left\|v_{\lambda}^{*}-v_{\lambda_{0}}^{*}\right\| \longrightarrow 0, \quad \text { as } \lambda \longrightarrow \lambda_{0} \text {. }
$$

(2) If

$$
\varphi(l) \geq \frac{1}{\varrho}\left(l^{1 / 2}-l\right)+l^{1 / 2}, \quad \forall l \in(0,1),
$$

then $0<\lambda_{1}<\lambda_{2}$ implies $v_{\lambda_{1}}^{*}<v_{\lambda_{2}}^{*}$.

(3) If there exists $\gamma \in(0,1 / 2)$ such that

$$
\varphi(l) \geq \frac{1}{\varrho}\left(l^{\gamma}-l\right)+l^{\gamma}, \quad \forall l \in(0,1),
$$

then

$$
\begin{array}{r}
\lim _{\lambda \longrightarrow 0^{+}} \sup _{t \in[0,1]}\left|v_{\lambda}^{*}(t)\right|=\lim _{\lambda \longrightarrow 0^{+}}\left\|v_{\lambda}^{*}\right\|=0, \\
\lim _{\lambda \longrightarrow+\infty} \sup _{t \in[0,1]}\left|v_{\lambda}^{*}(t)\right|=\lim _{\lambda \longrightarrow+\infty}\left\|v_{\lambda}^{*}\right\|=+\infty .
\end{array}
$$

\section{Numerical Examples}

In this section, we give two simple theoretical numerical examples which justify Theorems 1 and 2 .

Example 1. We consider the following equation:

$$
\begin{aligned}
{ }^{c} D_{0+}^{3 / 2} & \phi_{3 / 2}\left(D_{0+}^{29 / 10} v(t)\right)+t^{-(19 / 20)} v^{1 / 2}(t)+t^{-(9 / 20)} v^{1 / 2}(t) \\
& +2 t^{-(29 / 40)} v^{1 / 4}(t)\left(D_{0+}^{9 / 10} v(t)\right)^{1 / 4}+2 t^{19 / 20} v^{-(1 / 2)}(t) \\
& +2 t^{1 / 2}\left(D_{0+}^{9 / 10} v(t)\right)^{-(1 / 2)}=0, \quad 0<t<1
\end{aligned}
$$

with the boundary conditions 


$$
\begin{aligned}
v(0)= & D_{0+}^{10 / 11} v(0)=D_{0+}^{29 / 10} v(0)=\left(\phi_{3 / 2}\left(D_{0+}^{29 / 10} v(0)\right)\right)^{\prime}=0, \\
D_{0+}^{19 / 10} v(1)= & \frac{1}{100} \Gamma\left(\frac{3}{2}\right) \int_{0}^{1} s^{-(1 / 2)} D_{0+}^{7 / 5} v(s) d A_{1}(s) \\
& +\frac{1}{100} \int_{0}^{1} s^{-1} D_{0+}^{9 / 10} v(s) d A_{2}(s) \\
& +\frac{1}{100} \sum_{i=1}^{\infty} \frac{1}{i(i+1)} D_{0+}^{9 / 10} v(1),
\end{aligned}
$$

where $\phi_{3 / 2}(s)=|s|^{-(1 / 2)} s$ and

$$
\begin{aligned}
& A_{1}(t)= \begin{cases}\frac{2}{11}, & t \in[0,1) \\
\frac{13}{11}, & t=1,\end{cases} \\
& A_{2}(t)= \begin{cases}\frac{1}{13}, & t \in[0,1), \\
\frac{14}{13}, & t=1 .\end{cases}
\end{aligned}
$$

Proof. Let

$$
\begin{aligned}
f\left(t, u_{1}, u_{2}\right)= & t^{-(19 / 20)} u_{1}^{1 / 2}+t^{-(9 / 20)} u_{1}^{1 / 2}+2 t^{-(29 / 40)} u_{1}^{1 / 4} u_{2}^{1 / 4} \\
& +2 t^{19 / 20} u_{1}^{-(1 / 2)}+2 t^{1 / 2} u_{2}^{-(1 / 2)}
\end{aligned}
$$

$\phi_{p}(s)=\phi_{3 / 2}(s), \alpha=3 / 2, \beta=29 / 10, \beta_{1}=9 / 10, \nu_{1}=10 / 11$, $r_{0}=19 / 10, \quad r_{1}=7 / 5, \quad r_{2}=9 / 10, \quad r_{3}=9 / 10, \rho_{i}=1 / i(i+1)$, $\eta_{i}=1,(i=1,2, \ldots), \eta=1, \lambda_{1}=(1 / 100) \Gamma(3 / 2), \lambda_{2}=1 / 100$, $\lambda_{3}=1 / 100, \quad h_{1}(t)=t^{-(1 / 2)}$, and $h_{2}(t)=t^{-1}$. Then, the problem (124)-(125) can be expressed as the problem (4)-(5). Notice that

$$
\begin{aligned}
\int_{0}^{1} s^{\beta-r_{1}-1} h_{1}(s) d A_{1}(s)= & 1>0, \\
\int_{0}^{\eta} s^{\beta-r_{2}-1} h_{2}(s) d A_{2}(s)= & 1>0, \\
\sum_{i=1}^{\infty} \rho_{i} \eta_{i}^{\beta-r_{3}-1}= & 1, \\
\sigma= & \frac{1}{\Gamma\left(\beta-r_{0}\right)}-\frac{\lambda_{1}}{\Gamma\left(\beta-r_{1}\right)} \\
& \cdot \int_{0}^{1} s^{\beta-r_{1}-1} h_{1}(s) d A_{1}(s)-\frac{\lambda_{2}}{\Gamma\left(\beta-r_{2}\right)} \\
& \cdot \int_{0}^{\eta} s^{\beta-r_{2}-1} h_{2}(s) d A_{2}(s)-\frac{\lambda_{3}}{\Gamma\left(\beta-r_{3}\right)} \\
& \cdot \sum_{i=1}^{\infty} \rho_{i} \eta_{i}^{\beta-r_{3}-1}=0.97>0 .
\end{aligned}
$$

We infer that the properties of Green's function in Lemma 8 are achieved. Let

$$
\begin{aligned}
F\left(t, u_{1}, u_{2}, v_{1}, v_{2}\right)= & t^{-(19 / 20)} u_{1}^{1 / 2}+t^{-(29 / 40)} u_{1}^{1 / 4} u_{2}^{1 / 4} \\
& +t^{19 / 20} v_{1}^{-(1 / 2)}+t^{1 / 2} v_{2}^{-(1 / 2)} \\
G\left(t, u_{1}, u_{2}, v_{1}, v_{2}\right)= & t^{-(9 / 20)} u_{1}^{1 / 2}+t^{-(29 / 40)} u_{1}^{1 / 4} u_{2}^{1 / 4} \\
& +t^{19 / 20} v_{1}^{-(1 / 2)}+t^{1 / 2} v_{2}^{-(1 / 2)}
\end{aligned}
$$

It is easy to check the following conditions:

$\left(\mathbf{V}_{1}\right) f\left(t, u_{1}, u_{2}\right)=F\left(t, u_{1}, u_{2}, u_{1}, u_{2}\right)+G\left(t, u_{1}, u_{2}, u_{1}\right.$, $\left.u_{2}\right)$.

$\left(\mathbf{V}_{2}\right)$ For fixed $t \in(0,1)$, and $\left(v_{1}, v_{2}\right) \in(0,+\infty)^{2}$, $F\left(t, u_{1}, u_{2}, v_{1}, v_{2}\right), G\left(t, u_{1}, u_{2}, v_{1}, v_{2}\right)$ are increasing in $\left(u_{1}, u_{2}\right) \in(0,+\infty)^{2}$; for fixed $t \in(0,1)$ and $\left(u_{1}, u_{2}\right) \in$ $(0,+\infty)^{2}, F\left(t, u_{1}, u_{2}, v_{1}, v_{2}\right)$ and $G\left(t, u_{1}, u_{2}, v_{1}, v_{2}\right)$ are decreasing in $\left(v_{1}, v_{2}\right) \in(0,+\infty)^{2}$.

$\left(\mathbf{V}_{3}\right)$ Let $\varphi(l)=l$. Then, for $l \in(0,1), t \in(0,1)$, and $\left(u_{1}, u_{2}, v_{1}, v_{2}\right) \in(0,+\infty)^{4}$,

$$
\begin{aligned}
F( & \left.t, l u_{1}, l u_{2}, l^{-1} v_{1}, l^{-1} v_{2}\right) \\
= & t^{-(19 / 20)}\left(l u_{1}\right)^{1 / 2}+t^{-(29 / 40)}\left(l u_{1}\right)^{1 / 4}\left(l u_{2}\right)^{1 / 4} \\
& \quad+t^{19 / 20}\left(l^{-1} v_{1}\right)^{-(1 / 2)}+t^{1 / 2}\left(l^{-1} v_{2}\right)^{-(1 / 2)} \\
\geq & l^{1 / 2} F\left(t, u_{1}, u_{2}, v_{1}, v_{2}\right)=\varphi^{1 / 2}(l) F\left(t, u_{1}, u_{2}, v_{1}, v_{2}\right) \\
= & \varphi^{p-1}(l) F\left(t, u_{1}, u_{2}, v_{1}, v_{2}\right), \\
G( & \left.t, l u_{1}, l u_{2}, l^{-1} v_{1}, l^{-1} v_{2}\right) \\
= & t^{-(9 / 20)}\left(l u_{1}\right)^{1 / 2}+t^{-(29 / 40)}\left(l u_{1}\right)^{1 / 4}\left(l u_{2}\right)^{1 / 4} \\
& \quad+t^{19 / 20}\left(l^{-1} v_{1}\right)^{-(1 / 2)}+t^{1 / 2}\left(l^{-1} v_{2}\right)^{-(1 / 2)} \\
\geq & l^{1 / 2} G\left(t, u_{1}, u_{2}, v_{1}, v_{2}\right)=l^{p-1} G\left(t, u_{1}, u_{2}, v_{1}, v_{2}\right) .
\end{aligned}
$$

$\left(\mathbf{V}_{4}\right)$ Let $\varrho=1$. Then, for all $\left(u_{1}, u_{2}, v_{1}, v_{2}\right) \in(0,+\infty)^{4}$,

$$
F\left(t, u_{1}, u_{2}, v_{1}, v_{2}\right) \geq \varrho G\left(t, u_{1}, u_{2}, v_{1}, v_{2}\right) .
$$

$\left(\mathbf{V}_{5}\right)$ The functions $F$ and $G$ satisfy

$$
\begin{aligned}
0< & \int_{0}^{1}(1-\tau)^{\alpha-1} F\left(\tau, 1,1, \tau^{\beta-1}, \tau^{\beta-1}\right) d \tau \\
= & B(1.5,0.05)+B(1.5,0.275)+B(1.5,1) \\
& +B(1.5,0.55)<+\infty, \\
0< & \int_{0}^{1}(1-\tau)^{\alpha-1} G\left(\tau, 1,1, \tau^{\beta-1}, \tau^{\beta-1}\right) d \tau \\
= & B(1.5,0.55)+B(1.5,0.275)+B(1.5,1) \\
& +B(1.5,0.55)<+\infty .
\end{aligned}
$$



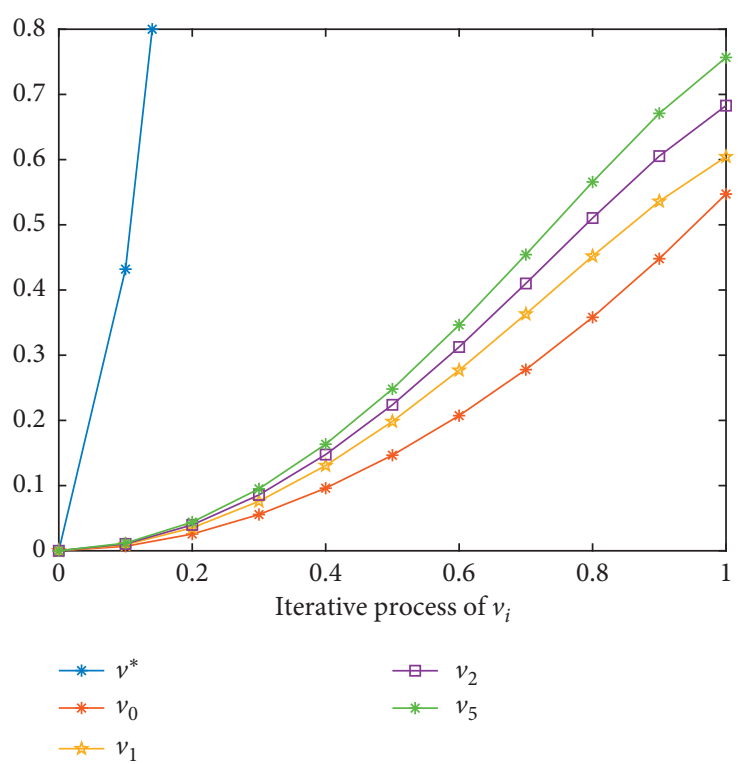

(a)

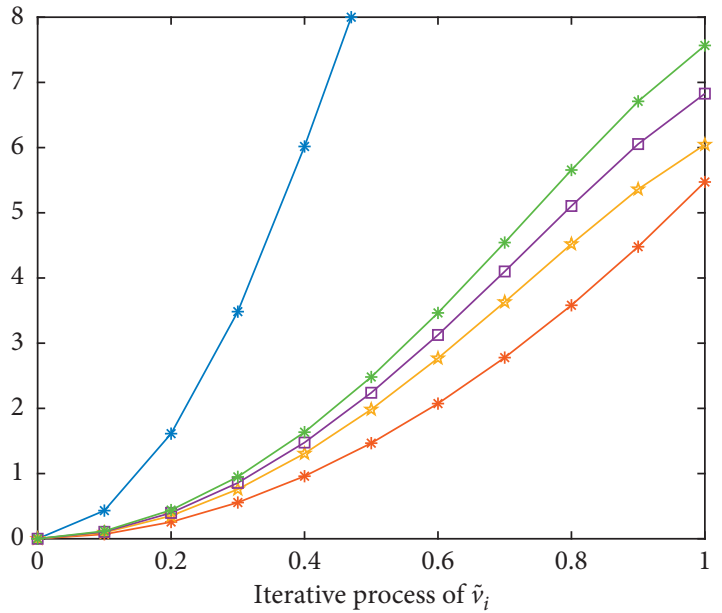

$\begin{array}{ll}\rightarrow & v^{*} \\ \rightarrow & \tilde{v}_{0} \\ \leftarrow & \tilde{v}_{1}\end{array}$

$\square \tilde{v}_{2}$

* $-\tilde{v}_{5}$

(b)

FIGURE 1: Iterative process of $v_{i}$ and $\widetilde{v}_{i}$.

Thus, the assumptions of Theorem 1 are satisfied. By calculation, we obtain the approximate solution of the problem $(124)-(125)$ is $v^{*}(t) \approx 34.315 t^{1.9}$. On the other hand, let $u_{0}(t)=t$ and $\widetilde{u}_{0}=10 t$. We take $v_{0}(t)=I^{\beta_{1}} u_{0}(t)$ and $\widetilde{v}_{0}=I^{\beta_{1}} \widetilde{u}_{0}(t)$. By iterating the sequences, the numerical results of the iterative process are shown as follows (Figure 1 and Tables 1 and 2).

Example 2. We consider the equation

$$
\begin{gathered}
{ }^{c} D_{0+}^{3 / 2} \phi_{3 / 2}\left(D_{0+}^{35 / 12} v(t)\right)+\lambda\left(2 t^{-(35 / 144)} v^{1 / 12}(t)\left(D_{0+}^{11 / 12} v(t)\right)^{1 / 12}\right. \\
\left.+2 t^{23 / 72} v^{-(1 / 6)}(t)+2 t^{1 / 6}\left(D_{0+}^{11 / 12} v(t)\right)^{-(1 / 6)}+1\right)=0
\end{gathered}
$$

with the boundary conditions

$$
\begin{aligned}
v(0)= & D_{0+}^{12 / 13} v(0)=D_{0+}^{35 / 12} v(0) \\
= & \left(\phi_{3 / 2}\left(D_{0+}^{35 / 12} v(0)\right)\right)^{\prime}=0, \\
D_{0+}^{23 / 12} v(1)= & \frac{1}{100} \Gamma\left(\frac{3}{2}\right) \int_{0}^{1} s^{-(1 / 2)} D_{0+}^{17 / 12} v(s) d A_{1}(s) \\
& +\frac{1}{100} \int_{0}^{1} s^{-1} D_{0+}^{11 / 12} v(s) d A_{2}(s) \\
& +\frac{1}{100} \sum_{i=1}^{\infty} \frac{1}{i(i+1)} D_{0+}^{11 / 12} v(1),
\end{aligned}
$$

TABLE 1: Iterative process of $v_{i}$.

\begin{tabular}{lccccccc}
\hline$t$ & $v_{0}$ & $v_{1}$ & $v_{2}$ & $v_{3}$ & $v_{4}$ & $v_{5}$ & $v_{*}$ \\
\hline 0.0 & 0.0000 & 0.0000 & 0.0000 & 0.0000 & 0.0000 & 0.0000 & 0.0000 \\
0.1 & 0.0069 & 0.0094 & 0.0106 & 0.0113 & 0.0116 & 0.0118 & 0.4320 \\
0.2 & 0.0257 & 0.0352 & 0.0397 & 0.0421 & 0.0433 & 0.0440 & 1.6123 \\
0.3 & 0.0556 & 0.0759 & 0.0857 & 0.0908 & 0.0935 & 0.0950 & 3.4835 \\
0.4 & 0.0960 & 0.1307 & 0.1476 & 0.1563 & 0.1610 & 0.1635 & 6.0172 \\
0.5 & 0.1466 & 0.1983 & 0.2239 & 0.2373 & 0.2443 & 0.2481 & 9.1945 \\
0.6 & 0.2073 & 0.2769 & 0.3126 & 0.3312 & 0.3411 & 0.3464 & 13.0008 \\
0.7 & 0.2779 & 0.3631 & 0.4100 & 0.4344 & 0.4474 & 0.4543 & 17.4249 \\
0.8 & 0.3581 & 0.4520 & 0.5104 & 0.5408 & 0.5570 & 0.5657 & 22.4572 \\
0.9 & 0.4480 & 0.5361 & 0.6054 & 0.6415 & 0.6606 & 0.6710 & 28.0895 \\
1.0 & 0.5472 & 0.6045 & 0.6828 & 0.7234 & 0.7450 & 0.7567 & 34.3150 \\
\hline
\end{tabular}

where $\phi_{3 / 2}(s)=|s|^{-(1 / 2)} s$ and

$$
\begin{aligned}
& A_{1}(t)= \begin{cases}\frac{2}{11}, & t \in[0,1), \\
\frac{13}{11}, & t=1,\end{cases} \\
& A_{2}(t)= \begin{cases}\frac{1}{13}, & t \in[0,1), \\
\frac{14}{13}, & t=1 .\end{cases}
\end{aligned}
$$

Proof. Let

$$
\begin{aligned}
& f\left(t, u_{1}, u_{2}\right)=\lambda\left(2 t^{-(35 / 144)} u_{1}^{1 / 12} u_{2}^{1 / 12}+2 t^{23 / 72} u_{1}^{-(1 / 6)}\right. \\
& \left.+2 t^{1 / 6} u_{2}^{-(1 / 6)}+1\right) \\
& \phi_{p}(s)=\phi_{3 / 2}(s), \alpha=3 / 2, \beta=35 / 12, \beta_{1}=11 / 12, v_{1}=12 / 13 \text {, } \\
& r_{0}=23 / 12, \quad r_{1}=17 / 12, \quad r_{2}=11 / 12, \quad r_{3}=11 / 12 \text {, }
\end{aligned}
$$


TABLE 2: Iterative process of $\widetilde{v}_{i}$.

\begin{tabular}{lccccccc}
\hline$t$ & $\widetilde{v}_{0}$ & $\widetilde{v}_{1}$ & $\widetilde{v}_{2}$ & $\widetilde{v}_{3}$ & $\widetilde{v}_{4}$ & $\widetilde{v}_{5}$ & $v_{*}$ \\
\hline 0.0 & 0.0000 & 0.0000 & 0.0000 & 0.0000 & 0.0000 & 0.0000 & 0.0000 \\
0.1 & 0.0689 & 0.0943 & 0.1064 & 0.1128 & 0.1161 & 0.1179 & 0.4320 \\
0.2 & 0.2571 & 0.3518 & 0.3971 & 0.4207 & 0.4333 & 0.4400 & 1.6123 \\
0.3 & 0.5555 & 0.7591 & 0.8570 & 0.9079 & 0.9350 & 0.9496 & 3.4835 \\
0.4 & 0.9596 & 1.3071 & 1.4757 & 1.5633 & 1.6100 & 1.6351 & 6.0172 \\
0.5 & 1.4663 & 1.9835 & 2.2394 & 2.3724 & 2.4433 & 2.4814 & 9.1945 \\
0.6 & 2.0733 & 2.7688 & 3.1262 & 3.3120 & 3.4109 & 3.4641 & 13.0008 \\
0.7 & 2.7788 & 3.6311 & 4.1000 & 4.3438 & 4.4735 & 4.5433 & 17.4249 \\
0.8 & 3.5814 & 4.5203 & 5.1043 & 5.4079 & 5.5695 & 5.6565 & 22.4572 \\
0.9 & 4.4796 & 5.3612 & 6.0542 & 6.4144 & 6.6062 & 6.7093 & 28.0895 \\
1.0 & 5.4724 & 6.0459 & 6.8277 & 7.2340 & 7.4502 & 7.5666 & 34.3150 \\
\hline
\end{tabular}

$\rho_{i}=1 / i(i+1), \quad \eta_{i}=1, \quad(i=1,2, \ldots), \quad \eta=1, \quad \lambda_{1}=$ $(1 / 100) \Gamma(3 / 2), \lambda_{2}=1 / 100, \lambda_{3}=1 / 100, h_{1}(t)=t^{-(1 / 2)}$, and $h_{2}(t)=t^{-1}$. Then, the problems (133)-(134) can be expressed as the problems (6)-(5). Notice that

$$
\begin{aligned}
\sigma= & \frac{1}{\Gamma\left(\beta-r_{0}\right)}-\frac{\lambda_{1}}{\Gamma\left(\beta-r_{1}\right)} \int_{0}^{1} s^{\beta-r_{1}-1} h_{1}(s) d A_{1}(s) \\
& -\frac{\lambda_{2}}{\Gamma\left(\beta-r_{2}\right)} \int_{0}^{\eta} s^{\beta-r_{2}-1} h_{2}(s) d A_{2}(s) \\
& -\frac{\lambda_{3}}{\Gamma\left(\beta-r_{3}\right)} \sum_{i=1}^{\infty} \rho_{i} \eta_{i}^{\beta-r_{3}-1} \\
= & 0.97>0 .
\end{aligned}
$$

We infer that the properties of Green's function in Lemma 8 are achieved. Let

$$
\begin{aligned}
F\left(t, u_{1}, u_{2}, v_{1}, v_{2}\right)= & \lambda\left(t^{-(35 / 144)} u_{1}^{1 / 12} u_{2}^{1 / 12}+t^{23 / 72} v_{1}^{-(1 / 6)}\right. \\
& \left.+t^{1 / 6} v_{2}^{-(1 / 6)}+1\right) \\
G\left(t, u_{1}, u_{2}, v_{1}, v_{2}\right)= & \lambda\left(t^{-(35 / 144)} u_{1}^{1 / 12} u_{2}^{1 / 12}+t^{23 / 72} v_{1}^{-(1 / 6)}\right. \\
& \left.+t^{1 / 6} v_{2}^{-(1 / 6)}\right)
\end{aligned}
$$

It is easy to check the conditions $\left(\mathbf{V}_{1}\right)-\left(\mathbf{V}_{5}\right)$ are all satisfied with $\varphi(l)=l^{1 / 3}$ and $\varrho=1$. On the other hand, let $\gamma=(4 / 9)<(1 / 2)$. Then,

$$
\begin{aligned}
& \text { (1) } \varphi(l)=l^{1 / 3} \geq(1 / \varrho)\left(l^{\gamma}-l\right)+l^{\gamma}=(6 / 5) l^{4 / 9}-(29 / 40) l, \\
& \forall l \in(0,1) \\
& \text { (2) } \varphi(l)=l^{1 / 3} \geq(1 / \varrho)\left(l^{1 / 2}-l\right)+l^{1 / 2}=(6 / 5) l^{1 / 2}-(29 / 40) l, \\
& \forall l \in(0,1)
\end{aligned}
$$

So, the assumptions of Theorem 2 are satisfied. In the following, we give the graphical simulations and table of the solution $v_{\lambda}^{*}$ with respect to $\lambda=0.5,1,1.5,2,3,10$, respectively (Figure 2 and Table 3 ).

Based on the graphical simulations and table, we obtain the following conclusions:

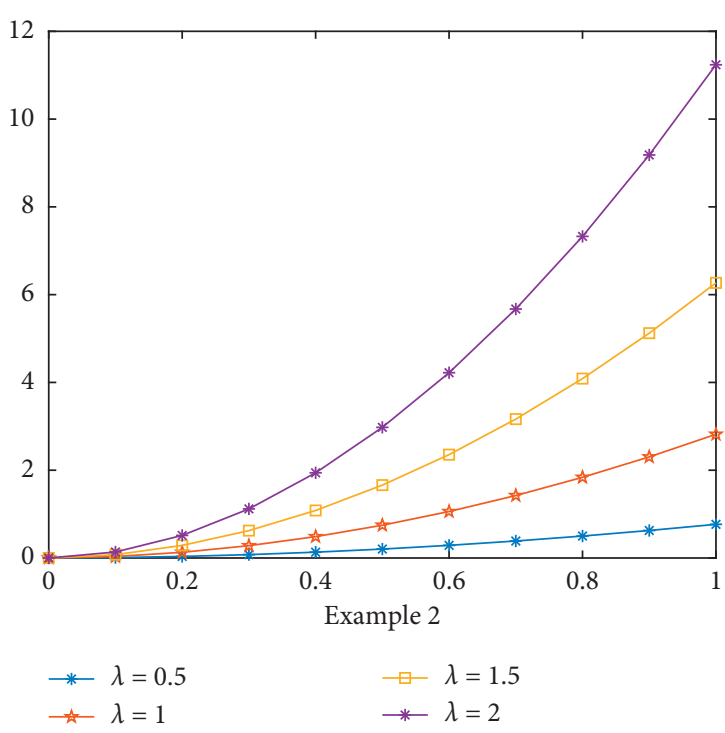

FIgURE 2: Dependence of the solution $v_{\lambda}^{*}$ upon $\lambda$.

TABle 3: Numerical value of the solution $v_{\lambda}^{*}$.

\begin{tabular}{lcccccc}
\hline$t$ & $\lambda=0.5$ & $\lambda=1$ & $\lambda=1.5$ & $\lambda=2$ & $\lambda=3$ & $\lambda=10$ \\
\hline 0.0 & 0.0000 & 0.0000 & 0.0000 & 0.0000 & 0.0000 & 0.0000 \\
0.1 & 0.0093 & 0.0342 & 0.0759 & 0.1362 & 0.3198 & 5.0454 \\
0.2 & 0.0351 & 0.1289 & 0.2867 & 0.5140 & 1.2073 & 19.0488 \\
0.3 & 0.0763 & 0.2805 & 0.6237 & 1.1182 & 2.6262 & 41.4358 \\
0.4 & 0.1324 & 0.4868 & 1.0825 & 1.9407 & 4.5582 & 71.9187 \\
0.5 & 0.2030 & 0.7466 & 1.6603 & 2.9765 & 6.9909 & 110.3027 \\
0.6 & 0.2880 & 1.0589 & 2.3548 & 4.2216 & 9.9151 & 156.4408 \\
0.7 & 0.3870 & 1.4229 & 3.1642 & 5.6727 & 13.3233 & 210.2155 \\
0.8 & 0.4998 & 1.8379 & 4.0871 & 7.3272 & 17.2093 & 271.5289 \\
0.9 & 0.6264 & 2.3034 & 5.1222 & 9.1830 & 21.5678 & 340.2972 \\
1.0 & 0.7666 & 2.8188 & 6.2684 & 11.2379 & 26.3942 & 416.4475 \\
\hline
\end{tabular}

(1) $v_{\lambda}^{*}$ is continuous with respect to $\lambda \in(0,+\infty)$. That is, for any $\lambda_{0} \in(0,+\infty)$,

$$
\sup _{t \in[0,1]}\left|v_{\lambda}^{*}(t)-v_{\lambda_{0}}^{*}(t)\right|=\left\|v_{\lambda}^{*}-v_{\lambda_{0}}^{*}\right\| \longrightarrow 0, \quad \text { as } \lambda \longrightarrow \lambda_{0} \text {. }
$$

(2) $0<\lambda_{1}<\lambda_{2}$ implies $v_{\lambda_{1}}^{*}<v_{\lambda_{2}}^{*}$.

(3) $\lim _{\lambda \longrightarrow 0^{+}} \sup _{t \in[0,1]}\left|v_{\lambda}^{*}(t)\right|=\lim _{\lambda \longrightarrow 0^{+}}\left\|v_{\lambda}^{*}\right\|=0, \lim _{\lambda \longrightarrow+\infty}$ $\sup _{t \in[0,1]}\left|v_{\lambda}^{*}(t)\right|=\lim _{\lambda \longrightarrow+\infty}\left\|v_{\lambda}^{*}\right\|=+\infty$.

\section{Conclusion}

In this paper, we introduce the fixed point theorem of mixed monotone operator for finding the uniqueness of positive solution of a class of fractional boundary value problems, which is a generalized form of turbulent flow problem in a porous medium. Two theoretical numerical examples are given to illustrate Theorems 1 and 2; the results then bring us a step closer to research the characters of solutions. 
Furthermore, as the application of mixed monotone operator operator, further research and discussion are required in practice.

\section{Data Availability}

The datasets used to support the findings of this study are included within the article.

\section{Conflicts of Interest}

The authors declare that they have no conflicts of interest.

\section{Acknowledgments}

The authors are supported financially by the National Natural Science Foundation of China (11871302) and the Natural Science Foundation of Shandong Province of China (ZR2017MA036). The support from the Australian Research Council for the research is also acknowledged.

\section{References}

[1] D. Baleanu, K. Diethelm, E. Scalas, and J. J. Trujillo, Fractional Calculus: Models and Numerical Methods, Series on Complexity, Nonlinearity and Chaos, World Scientific, Boston, MA, USA, 2012.

[2] A. S. Perelson and P. W. Nelson, "Mathematical analysis of HIV-1 dynamics in vivo," SIAM Review, vol. 41, no. 1, pp. 3-44, 1999.

[3] Y. Wang, L. Liu, X. Zhang, and Y. Wu, "Positive solutions of an abstract fractional semipositone differential system model for bioprocesses of HIV infection," Applied Mathematics and Computation, vol. 258, pp. 312-324, 2015.

[4] A. A. Kilbas, H. M. Srivastava, and J. J. Trujillo, "Theory and applications of fractional differential equations," in NorthHolland Mathematics Studies, Vol. 204, Elsevier Science B.V., Amsterdam, Netherlands, 2006.

[5] V. Lakshmikantham and A. S. Vatsala, "Basic theory of fractional differential equations," Nonlinear Analysis: Theory, Methods \& Applications, vol. 69, no. 8, pp. 2677-2682, 2008.

[6] I. Podlubny, Fractional Differential Equations, Mathematics in Science and Engineering, Academic Press, New York, NY, USA, 1999.

[7] Z. Bai and H. Lü, "Positive solutions for boundary value problem of nonlinear fractional differential equation," Journal of Mathematical Analysis and Applications, vol. 311, no. 2, pp. 495-505, 2005.

[8] S. G. Samko, A. A. Kilbas, and O. I. Marichev, Fractional Integrals and Derivatives: Theory and Applications, Gordon \& Breach, Yverdon, Switzerland, 1993.

[9] J. Henderson and R. Luca, Boundary Value Problems for Systems of Differential, Difference and Fractional Equations: Positive Solutions, Elsevier, Amsterdam, Netherlands, 2016.

[10] D. Guo, Y. J. Cho, and J. Zhu, Partial Ordering Methods in Nonlinear Problems, Nova Science Publishers, Inc., Hauppauge, NY, USA, 2004.

[11] D. Guo and V. Lakshmikantham, "Coupled fixed points of nonlinear operators with applications," Nonlinear Analysis: Theory, Methods \& Applications, vol. 11, no. 5, pp. 623-632, 1987.

[12] D. Guo and V. Lakshmikantham, Nonlinear Problems in Abstract Cones, Academic Press, San Diego, CA, USA, 1988.
[13] L. Liu, X. Zhang, J. Jiang, and Y. Wu, "The unique solution of a class of sum mixed monotone operator equations and its application to fractional boundary value problems," Journal of Nonlinear Sciences and Applications, vol. 9, no. 5, pp. 29432958, 2016.

[14] X. Zhang, "Positive solutions for a class of singular fractional differential equation with infinite-point boundary value conditions," Applied Mathematics Letters, vol. 39, pp. 22-27, 2015.

[15] J. Xu and Z. Wei, "Positive solutions for a class of fractional boundary value problems," Nonlinear Analysis: Modelling and Control, vol. 21, no. 1, pp. 1-17, 2016.

[16] X. Liu, M. Jia, and W. Ge, "The method of lower and upper solutions for mixed fractional four-point boundary value problem with $p$-Laplacian operator," Applied Mathematics Letters, vol. 65, pp. 56-62, 2017.

[17] X. Zhang, L. Liu, and Y. Wu, "Existence and uniqueness of iterative positive solutions for singular Hammerstein integral equations," The Journal of Nonlinear Sciences and Applications, vol. 10, no. 7, pp. 3364-3380, 2017.

[18] X. Zhang, L. Liu, B. Wiwatanapataphee, and Y. Wu, "The eigenvalue for a class of singular $p$-Laplacian fractional differential equations involving the Riemann-Stieltjes integral boundary condition," Applied Mathematics and Computation, vol. 235, pp. 412-422, 2014.

[19] J. Jiang, L. Liu, and Y. Wu, "Positive solutions for $p$-Laplacian fourth-order differential system with integral boundary conditions," Discrete Dynamics in Nature and Society, vol. 2012, Article ID 293734, 19 pages, 2012.

[20] Y. Tian, S. Sun, and Z. Bai, "Positive solutions of fractional differential equations with $p$-Laplacian," Journal of Function Spaces, vol. 2017, Article ID 3187492, 9 pages, 2017.

[21] L. Guo, L. Liu, and Y. Wu, "Iterative unique positive solutions for singular $p$-Laplacian fractional differential equation system with several parameters," Nonlinear Analysis: Modelling and Control, vol. 23, no. 2, pp. 182-203, 2018.

[22] L. Liu, H. Li, C. Liu, and Y. Wu, "Existence and uniqueness of positive solutions for singular fractional differential systems with coupled integral boundary conditions," The Journal of Nonlinear Sciences and Applications, vol. 10, no. 1, pp. 243262, 2017.

[23] D. Qarot, B. Ahmad, and A. Alsaedi, "Existence theorems for semi-linear Caputo fractional differential equations with nonlocal discrete and integral boundary conditions," Fractional Calculus and Applied Analysis, vol. 19, no. 2, pp. 463-479, 2016.

[24] D. Min, L. Liu, and Y. Wu, "Uniqueness of positive solutions for the singular fractional differential equations involving integral boundary value conditions," Boundary Value Problems, vol. 2018, no. 1, 2018.

[25] X. Liu, L. Liu, and Y. Wu, "Existence of positive solutions for a singular nonlinear fractional differential equation with integral boundary conditions involving fractional derivatives," Boundary Value Problems, vol. 2018, no. 1, 2018.

[26] X. Zhang and Q. Zhong, "Uniqueness of solution for higherorder fractional differential equations with conjugate type integral conditions," Fractional Calculus and Applied Analysis, vol. 20, no. 6, pp. 1471-1484, 2017.

[27] X. Zhang and Q. Zhong, "Triple positive solutions for nonlocal fractional differential equations with singularities both on time and space variables," Applied Mathematics Letters, vol. 80, pp. 12-19, 2018.

[28] Y. Cui, "Uniqueness of solution for boundary value problems for fractional differential equations," Applied Mathematics Letters, vol. 51, pp. 48-54, 2016. 
[29] C. S. Goodrich, "Existence of a positive solution to a class of fractional differential equations," Applied Mathematics Letters, vol. 23, no. 9, pp. 1050-1055, 2010.

[30] B. Zhu, L. Liu, and Y. Wu, "Local and global existence of mild solutions for a class of semilinear fractional integro-differential equations," Fractional Calculus and Applied Analysis, vol. 20, no. 6, pp. 1338-1355, 2017.

[31] C. Zhai and L. Wang, "Some existence, uniqueness results on positive solutions for a fractional differential equation with infinite-point boundary conditions," Nonlinear Analysis: Modelling and Control, vol. 22, no. 4, pp. 566-577, 2017.

[32] L. Liu, X. Zhang, L. Liu, and Y. Wu, "Iterative positive solutions for singular nonlinear fractional differential equation with integral boundary conditions," Advances in Difference Equations, vol. 2016, no. 1, p. 154, 2016.

[33] J. Henderson and R. Luca, "Existence of nonnegative solutions for a fractional integro-differential equation," Results in Mathematics, vol. 72, no. 1-2, pp. 747-763, 2017.

[34] L. Guo, L. Liu, and Y. Wu, "Uniqueness of iterative positive solutions for the singular fractional differential equations with integral boundary conditions," Boundary Value Problems, vol. 2016, no. 1, p. 147, 2016.

[35] J. Wu, X. Zhang, L. Liu, and Y. Wu, "Twin iterative solutions for a fractional differential turbulent flow model," Boundary Value Problems, vol. 2016, no. 1, 2016.

[36] J. Wu, X. Zhang, L. Liu, Y. Wu, and B. Wiwatanapataphee, "Iterative algorithm and estimation of solution for a fractional order differential equation," Boundary Value Problems, vol. 2016, no. 1, 2016.

[37] X. Hao, H. Wang, L. Liu, and Y. Cui, "Positive solutions for a system of nonlinear fractional nonlocal boundary value problems with parameters and p-Laplacian operator," Boundary Value Problems, vol. 2017, no. 1, 2017.

[38] T. Ren, S. Li, X. Zhang, and L. Liu, "Maximum and minimum solutions for a nonlocal $p$-Laplacian fractional differential system from eco-economical processes," Boundary Value Problems, vol. 2017, no. 1, 2017.

[39] R. Luca, "Positive solutions for a system of fractional differential equations with $p$-Laplacian operator and multi-point boundary conditions," Nonlinear Analysis: Modelling and Control, vol. 23, no. 5, pp. 771-801, 2018.

[40] B. Ahmad and R. Luca, "Existence of solutions for sequential fractional integro-differential equations and inclusions with nonlocal boundary conditions," Applied Mathematics and Computation, vol. 339, pp. 516-534, 2018.

[41] B. Ahmad and R. Luca, "Existence of solutions for a system of fractional differential equations with coupled nonlocal boundary conditions," Fractional Calculus and Applied Analysis, vol. 21, no. 2, pp. 423-441, 2018.

[42] J. Henderson and R. Luca, "Systems of Riemann-Liouville fractional equations with multi-point boundary conditions," Applied Mathematics and Computation, vol. 309, pp. 303-323, 2017.

[43] X. Hao, L. Zhang, and L. Liu, "Positive solutions of higher order fractional integral boundary value problem with a parameter," Nonlinear Analysis: Modelling and Control, vol. 24, no. 2, pp. 210-223, 2019.

[44] F. Wang, L. Liu, D. Kong, and Y. Wu, "Existence and uniqueness of positive solutions for a class of nonlinear fractional differential equations with mixed-type boundary value conditions," Nonlinear Analysis: Modelling and Control, vol. 24, no. 1, pp. 73-94, 2019.

[45] X. Hao, M. Zuo, and L. Liu, "Multiple positive solutions for a system of impulsive integral boundary value problems with sign-changing nonlinearities," Applied Mathematics Letters, vol. 82, pp. 24-31, 2018.

[46] X. Zhang, L. Liu, Y. Wu, and B. Wiwatanapataphee, "The spectral analysis for a singular fractional differential equation with a signed measure," Applied Mathematics and Computation, vol. 257, pp. 252-263, 2015.

[47] G. Wang, D. Baleanu, and L. Zhang, "Monotone iterative method for a class of nonlinear fractional differential equations," Fractional Calculus and Applied Analysis, vol. 15, no. 2, pp. 244-252, 2012.

[48] L. S. Leibenson, "General problem of the movement of a compressible fluid in a porous medium," Izvestiya Akademii Nauk Kirgizskoi SSR, vol. 9, pp. 7-10, 1983, in Russian.

[49] D. Jiang and W. Gao, "Upper and lower solution method and a singular boundary value problem for the one-dimensional $p$ Laplacian," Journal of Mathematical Analysis and Applications, vol. 252, no. 2, pp. 631-648, 2000.

[50] T. Chen, W. Liu, and C. Yang, "Antiperiodic solutions for Liénard-type differential equation with $p$-Laplacian operator," Boundary Value Problems, vol. 2010, no. 1, Article ID 194824, 2010.

[51] L. Guo and L. Liu, "Unique iterative positive solutions for singular $p$-Laplacian fractional differential equation system with infinite-point boundary conditions," Boundary Value Problems, vol. 2019, no. 1, 2019.

[52] F. Wang, L. Liu, and Y. Wu, "Iterative unique positive solutions for a new class of nonlinear singular higher order fractional differential equations with mixed-type boundary value conditions," Journal of Inequalities and Applications, vol. 2019, no. 1, 2019.

[53] K. Pei, G. Wang, and Y. Sun, "Successive iterations and positive extremal solutions for a Hadamard type fractional integro-differential equations on infinite domain," Applied Mathematics and Computation, vol. 312, pp. 158-168, 2017.

[54] G. Wang, K. Pei, R. P. Agarwal, L. Zhang, and B. Ahmad, "Nonlocal Hadamard fractional boundary value problem with Hadamard integral and discrete boundary conditions on a half-line," Journal of Computational and Applied Mathematics, vol. 343, pp. 230-239, 2018. 


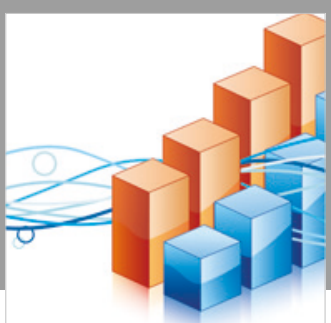

Advances in

Operations Research

\section{-n-m}
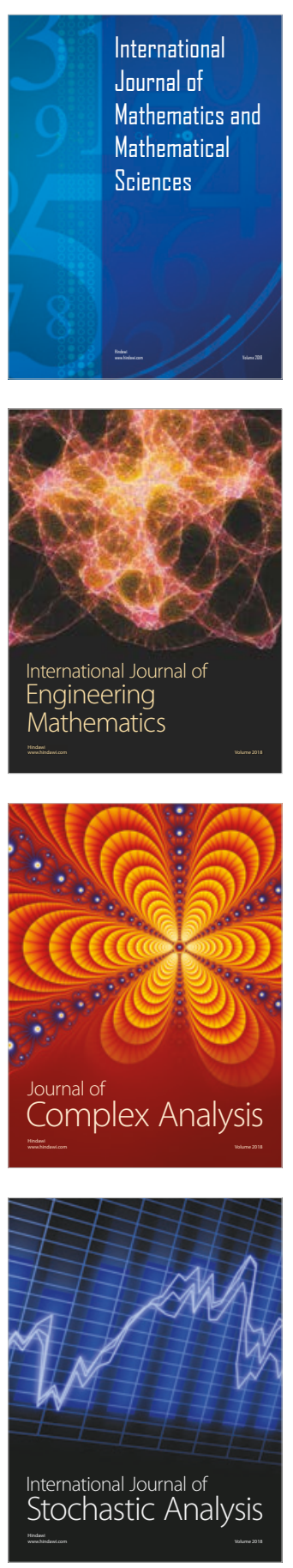
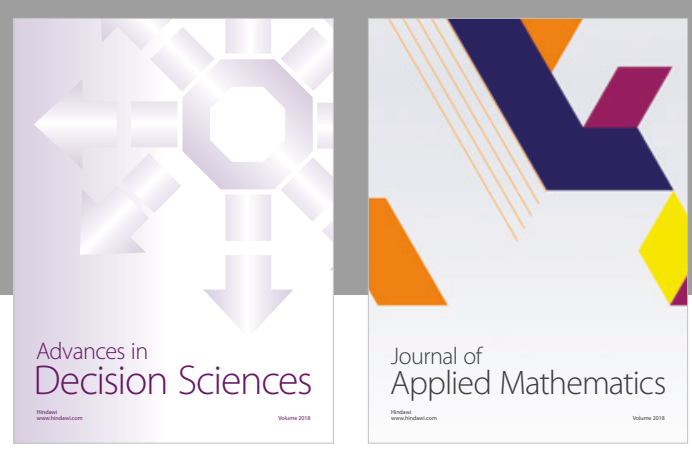

Journal of

Applied Mathematics
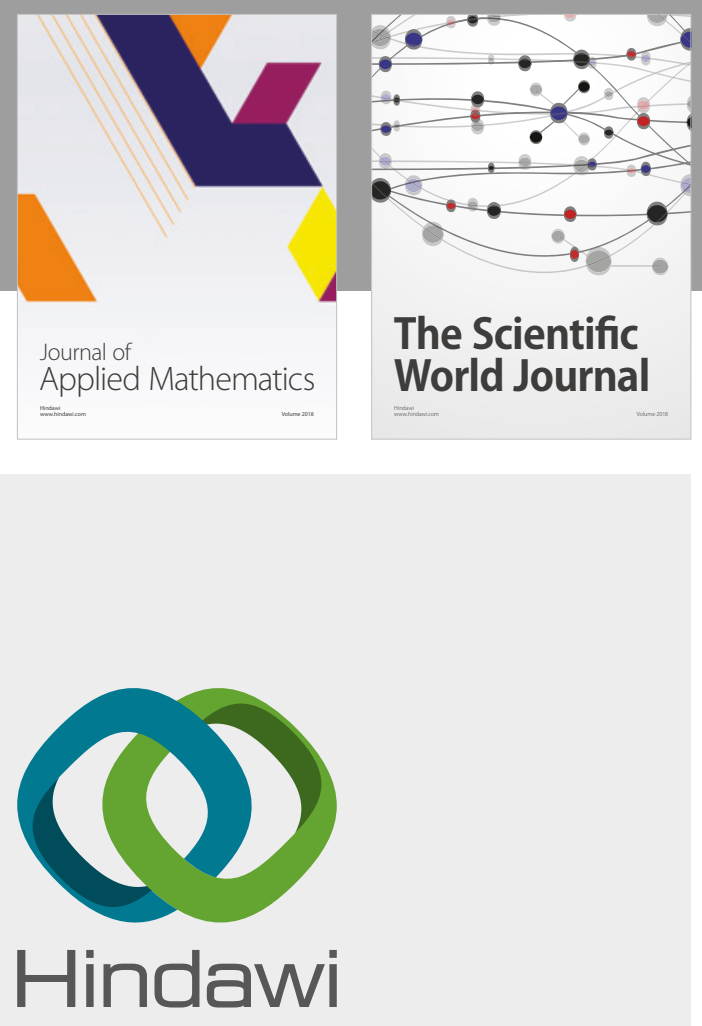

Submit your manuscripts at

www.hindawi.com

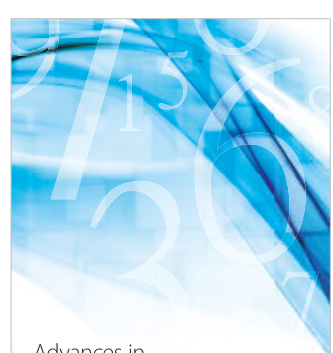

Advances in
Numerical Analysis
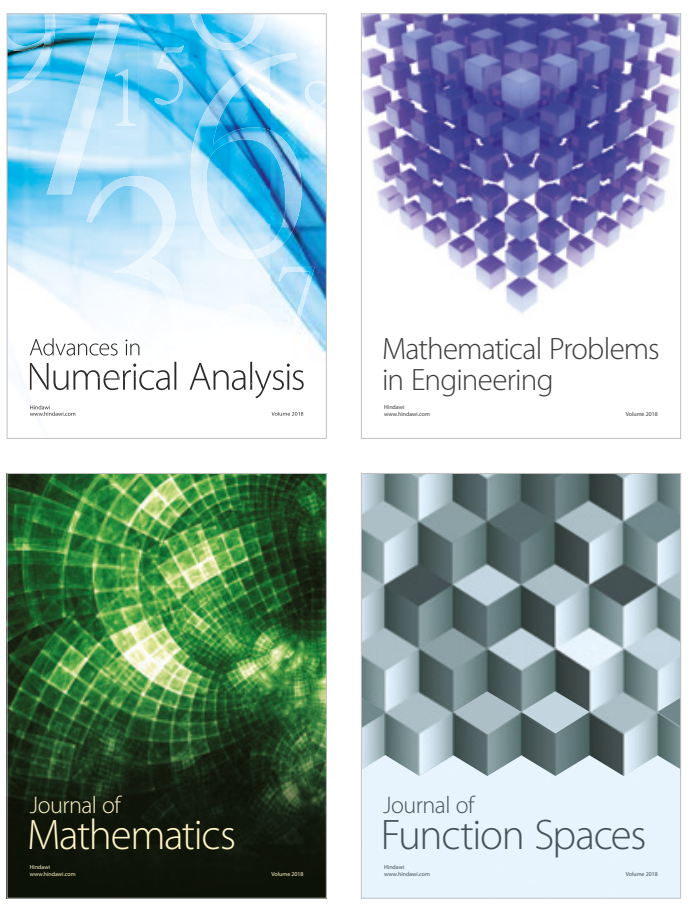

Mathematical Problems in Engineering

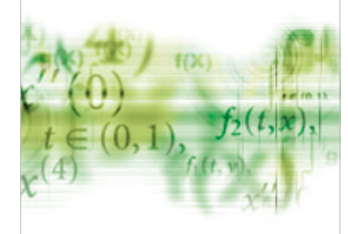

International Journal of

Differential Equations

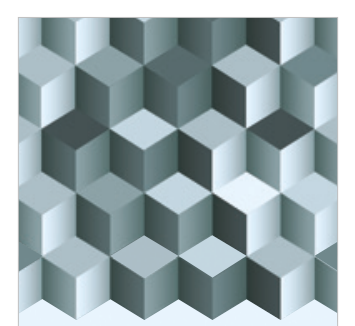

Journal of

Function Spaces
The Scientific

World Journal

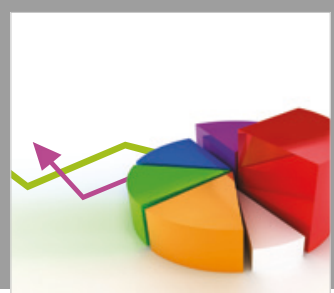

Journal of

Probability and Statistics
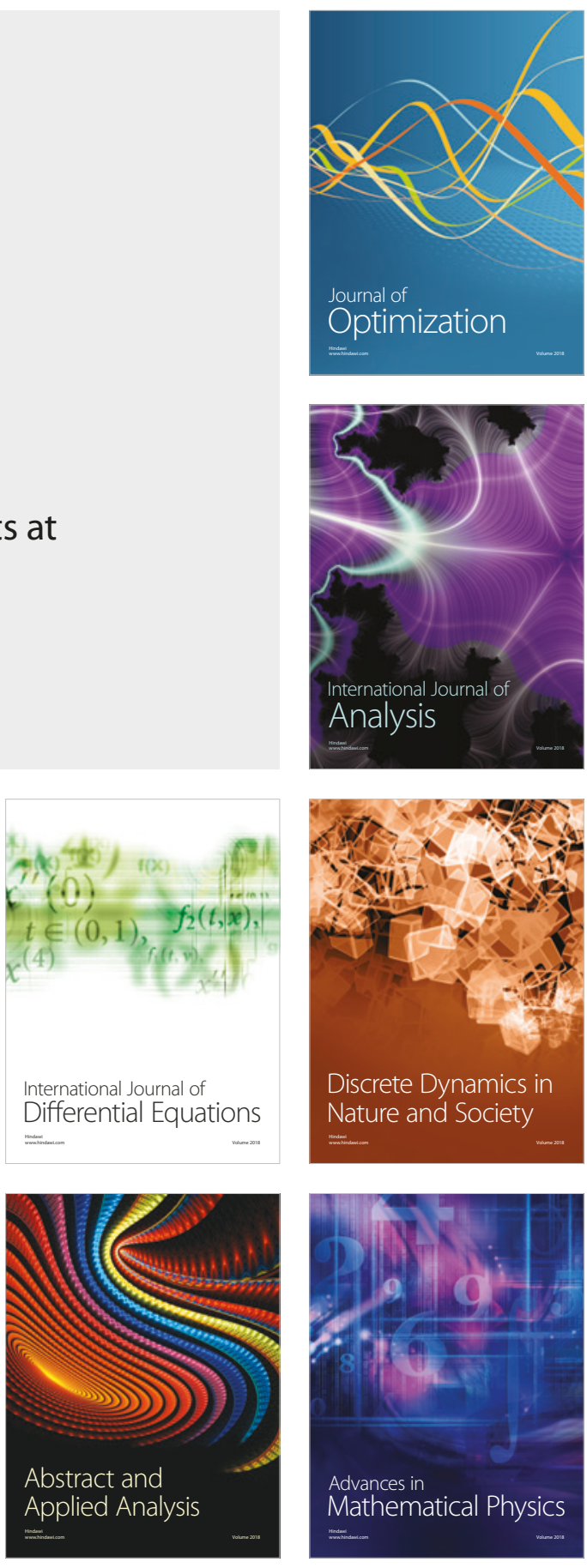